IPMU11-0053

\title{
Holographic Conductivity in Disordered Systems
}

\author{
Shinsei Ryu ${ }^{a}$ 円, Tadashi Takayanagi $b$ q, and Tomonori Ugajin ${ }^{b}$ \\ ${ }^{a}$ Department of Physics, University of California, Berkeley, CA 94720, USA \\ ${ }^{b}$ Institute for the Physics and Mathematics of the Universe (IPMU), \\ University of Tokyo, Kashiwa, Chiba 277-8582, Japan
}

\begin{abstract}
The main purpose of this paper is to holographically study the behavior of conductivity in $2+1$ dimensional disordered systems. We analyze probe D-brane systems in AdS/CFT with random closed string and open string background fields. We give a prescription of calculating the DC conductivity holographically in disordered systems. In particular, we find an analytical formula of the conductivity in the presence of codimension one randomness. We also systematically study the AC conductivity in various probe brane setups without disorder and find analogues of Mott insulators.
\end{abstract}

\footnotetext{
${ }^{1}$ e-mail:sryu@berkeley.edu

${ }^{2}$ e-mail:tadashi.takayanagi@ipmu.jp

${ }^{3}$ e-mail:tomoki.ugajin@ipmu.jp
} 


\section{Contents}

1 Introduction

2 Holographic Conductivity in Probe D-branes 4

2.1 General Setup . . . . . . . . . . . . . . . . .

2.2 Calculating Holographic Conductivity . . . . . . . . . . . . . . 6

2.3 DC Conductivity . . . . . . . . . . . . . . . . 8

3 AC Conductivity: From Metals to Insulators 10

3.1 Charged Probe Brane in AdS: Holographic Metal . . . . . . . . . . . . . . 10

3.2 Probe Brane vs. Bulk Approach in Holographic Metals and Superconductors 12

3.3 Holographic Metals with Pseudo Gaps . . . . . . . . . . . . . . . . . 13

3.4 Towards Holographic Mott Insulators . . . . . . . . . . . . . . . . . . . . 16

4 Holographic Conductivity in Disordered Systems 20

4.1 Origins of Randomness in Probe Brane Setup . . . . . . . . . . . . . . . . 20

4.2 Definition of Conductivity in Disordered Systems . . . . . . . . . . . . . . 21

4.3 Analysis of Equations of Motion . . . . . . . . . . . . . . . 22

4.4 Boundary Conditions . . . . . . . . . . . . . . . . . . . . . 23

4.5 Calculation of DC Conductivity . . . . . . . . . . . . . 24

4.6 Random Dilaton: Charge Neutral Impurities . . . . . . . . . . . . . . . . . 26

4.7 Random Charge Density: Charged Impurities . . . . . . . . . . . . . . . 27

4.8 Random Holes on Horizon . . . . . . . . . . . . . . . . . . . . . . . . . 28

4.9 Codimension Two Randomness . . . . . . . . . . . . . . . . . 31

5 Conclusions and Discussions 35

A Sum Rule 37 


\section{Introduction}

The AdS/CFT correspondence [1, 2, 3, 4] has been a powerful tool to investigate strongly coupled condensed matter systems because it claims that a strongly coupled gauge theory is equivalent to a classical gravity theory which lives in higher dimensions [5, 6, 7, 8, 9. Until now, such applications include, for example, quantum entanglement [10, 7], superconductors 111, 12, 5, 6], non-relativistic systems 13, 14, 15, non-fermi liquids [16, 17], the quantum Hall effect [18, 19, 20] and topological insulators [21, 22].

Another important problem in realistic condensed matter systems is the effect of quenched disorder due to impurities. For example, when sufficiently strong, disorder by itself can turn extended electron wavefunctions in a metal into spatially localized ones and thus triggers a metal-to-insulator transition - phenomenon known as Anderson localization and Anderson meta-insulator transition [23, 24] (for reviews see, e.g., [25, 26, 27]).

In many realistic metal-insulator transition scenarios, not only disorder but also electronelectron interactions come into play, and we must study the interplay of disorder and interactions. The systems of most experiential interest include phosphorus-doped silicon, the plateau transition in the quantum Hall effect, and the observation of a possible metal-insulator transition in two dimensions 28]. Graphene, a two-dimensional sheet of graphite, is another system where the combined effect of disorder and interactions play a crucial role 29].

However, dealing with systems with both strong disorder and strong interactions is to a large extent unsolved problem in condensed matter physics; surprisingly little is understood theoretically in the fully interacting disordered problem. It is therefore very interesting to see how the impurity effect looks like in the holographic approach. This enables us to understand how strongly coupled systems behave in the presence of randomness. An analysis of momentum relaxation in the presence of weak disorder has been done in [30]. A holographic replica method for arbitrary strength of the randomness has been formulated in [31]. Impurity lattices have been holographically constructed using Dbranes in [32]. A functional renormalization scheme [33] has been developed in 34] from a holographic viewpoint. In spite of these developments, however, holographic calculations of transport coefficients such as conductivity have not been established yet (nevertheless see [35] for a progress in this direction using the replica method). In this paper we will present a general framework to compute holographic conductivity in quenched disordered systems, based on the probe D-brane approach in AdS/CFT [36, 37.

The probe D-brane analysis has two important advantages. One is that we can neglect the backreactions to closed string fields. The other is that we can obtain the conductivity 
in the presence of randomness even from classical calculations. In the bulk approach based on a random perturbation of scalar operators, the disorder only affects the conductivity at one loop or higher loops in the gravity dual [31, 35]. Generally speaking, the holographic dual of a probe D-brane in AdS space is given by a Yang-Mills theory with quarks. The quarks are present in a flavor sector 36] or defect sector 38 and they are coupled to gluons and other matter fields which belong to the adjoint representation. As usual in this subject, we regard the charge under the $U(1)$ flavor symmetry as the electric charge of electrons, while the non-abelian $S U(N)$ gauge symmetry of the Yang-Mills theory is interpreted as the emergent gauge symmetry in condensed matter systems. An electron is regarded as a gauge invariant operator with the flavor charge. Notice that even though there is no dynamical $U(1)$ gauge field of photons, the emergent $S U(N)$ gauge interaction can be regarded as some analogue of the interactions due to the coulomb force and phonons. If we concentrate on the probe D-brane and forget about its backreaction to gravity sector, this corresponds to an open system of quarks interacting with outer systems of gluons, which looks analogous to electrons in a phonon bath. In this sense, it is not so unnatural to speculate that the strongly correlated electrons in condensed matter systems are described by strongly coupled gauge theories with quarks. Throughout this paper we will mainly concentrate on the setups dual to $2+1$ dimensional systems, though its higher dimensional generalization can be done similarly.

In the first half of this paper, we give a systematic summary of the holographic calculation of AC and DC conductivity in general setups of probe branes without introducing the randomness. The calculation of conductivity in probe D-branes has been done first in [37]. For other earlier studies in this direction see e.g. [39, 40, 41, 42, 43, 44]. We present several new numerical results of AC conductivity, which behave like various states of condensed matter systems from metals to insulators. In addition to the Anderson insulators, metals can be changed into insulators via another mechanism which is induced by strong interactions between electrons. This is known as Mott insulators 45. The insulators we find in the holographic setups look analogous to Mott insulators in that the strong coupling effect is very important.

The latter half of this paper will devoted to the analysis of holographic conductivity in disordered systems. We take into account the disorder effects both from the random fluctuations of the $U(1)$ gauge field on the brane and those of the supergravity backgrounds of the probe brane. We obtain an analytical formula of the holographic DC conductivity in disordered systems for a codimension one randomness as well as perturbative results in more general cases of randomness. We will also see that depending on the distributions of the impurities we can interpolate between metallic and insulating phases. 
This paper is organized as follows. In section two, we study general setups of probe D-branes and calculate the $\mathrm{AC}$ and $\mathrm{DC}$ conductivity. In section three, we present several explicit examples of calculations of the AC conductivity, which are regarded as holographic metals and insulators. In section four, we study the DC conductivity in the presence of various randomness. In section five, we summarize our conclusions and discuss future problems.

\section{Holographic Conductivity in Probe D-branes}

\subsection{General Setup}

We start with a probe $\mathrm{D} p$-brane extended in the $3+1$ dimensions with the other $p-3$ dimensions compactified on a cycle $M_{p-3}$ in a string theory background. We will work within a supergravity approximation and for an appropriate background with its holographic dual, this probe brane is dual to a quark-like flavor degrees of freedom coupled with a bulk gluon sector [36. This may be intuitively thought to be analogous to the electron systems (dual to the probe brane) in a phonon bath (dual to the bulk supergravity) [37.

The non-compact coordinate of the brane is denoted by $(t, x, y, z)$. The supergravity background is specified by the following metric in the Einstein frame

$$
d s_{E i n}^{2}=R^{2}\left(\frac{d z^{2}}{z^{2} f(z)}-\frac{h(z)}{z^{2}} d t^{2}+\frac{d x^{2}+d y^{2}}{z^{2}}\right)+d s_{6}^{2},
$$

and the string coupling constant given in terms of $z$ dependent dilaton

$$
g_{s}=g_{s}^{(0)} \cdot e^{\phi(z)} .
$$

For various examples of such dilatonic solutions refer to [46, 43]. We shift that the value of the dilaton and define $g_{s}^{(0)}$ such that it vanishes as $\phi(0)=0$ at the boundary $z=0$ of the supergravity background (2.1). Notice that the six dimensional space described by the metric $d s_{6}^{2}$ is not necessarily compact; it can include non-compact coordinates in which the $\mathrm{D} p$-brane is not extended. For example, a probe D3-brane can extend in the direction of $\mathrm{AdS}_{4}$ in a $\mathrm{AdS}_{5}$ spacetime. On the other hand, $d s_{6}^{2}$ also includes a compact $p-3$ dimensional cycle $M_{p-3}$ on which the $\mathrm{D} p$-brane is wrapped. The string frame metric $G_{S}$ is related to the Einstein frame metric $G_{E}$ via $G_{E}=e^{-\phi / 2} G_{S}$. For gravity duals of the finite temperature systems, the functions $f(z)$ and $h(z)$ have a zero at the horizon $z=z_{H}$. The temperature $T$ is inversely proportional to $z_{H}$ as usual. In this paper we 
assume that the four dimensional spacetime spanned by $(t, x, y, z)$ is asymptotically AdS, though we can generalize our analysis to higher dimensions without difficulty.

The DBI action of a probe $\mathrm{D} p$-brane looks like f

$$
\begin{aligned}
& S_{D B I}^{D p}=-\tau_{p} \int(d \xi)^{p+1} e^{-\varphi(z)} \sqrt{-\operatorname{det}\left(G_{S}+F\right)} \\
& =-\frac{T_{p}}{\left(2 \pi \alpha^{\prime}\right)^{2}} \int d t d x d y d z e^{-\varphi(z)} \sqrt{-\operatorname{det}\left(G_{E} e^{\phi / 2}+F\right)}
\end{aligned}
$$

where $\xi$ denotes the $p+1$ coordinates in which the brane extends. The field strength of the $U(1)$ gauge field on the brane is denoted by $F=d A$. The constant $\tau_{p}=\frac{1}{g_{s}^{(0)}(2 \pi)^{p}\left(\alpha^{\prime}\right)^{(p+1) / 2}}$ is the standard tension of a $\mathrm{D} p$-brane with the string coupling constant evaluated at the boundary $z=0 . \varphi(z)$ depends both on the dilaton and the volume of $M_{p-3}$. More explicitly, we have

$$
T_{p} \cdot e^{-\varphi(z)}=\left(2 \pi \alpha^{\prime}\right)^{2} \cdot \tau_{p} \cdot V_{p-3}(z) \cdot e^{-\phi(z)},
$$

where $V_{p-3}(z)$ is the $z$ dependent volume of $M_{p-3}$ in string frame. We fix the constant part of $\varphi(z)$ by the condition $\varphi(0)=0$. In particular, if $V_{p-3}$ does not depend on $z$, then we simply find $\varphi(z)=\phi(z)$. Notice that $T_{p}$ is normalized such that it is dimensionless.

Explicitly we can evaluate the DBI action as follows

$$
S_{D B I}=-\frac{R^{2} T_{p}}{\left(2 \pi \alpha^{\prime}\right)^{2}} \int \frac{d t d x d y d z}{z^{4}} e^{-\varphi+\phi} \sqrt{\frac{h R^{4}}{f}-e^{-\phi} z^{4} F_{t z}^{2}-\frac{z^{4} e^{-\phi}}{f} F_{t x}^{2}+z^{4} h e^{-\phi} F_{z x}^{2}} .
$$

In this paper, we will work in the gauge $A_{z}=0$,

In order to have a holographic dual with non-zero charged density, we need a solution with the electric flux $F_{t z}$. This is given by

$$
A_{t}(z)=\mu+\rho \int_{0}^{z} d y \sqrt{\frac{h(y)}{f(y) F(y)}}
$$

where we defined

$$
F(z)=e^{-2 \varphi(z)}+\frac{\rho^{2}}{R^{4}} z^{4} e^{-\phi(z)}
$$

Notice that in our normalization, i.e. $\phi(0)=\varphi(0)=0$, we always find $F(0)=1$ at the boundary.

${ }^{4}$ The effective action of a D-brane also includes Chern-Simons terms which express couplings to RR fields. However, in this paper we only consider probe brane configurations where we can neglect such terms. The Chern-Simons terms become important when we are interested in topological properties as observed in the quantum Hall effect [18, 19, 20] and topological insulators [21, 22]. 
According to the standard bulk to boundary relation in gravity duals, in our model (2.6) the charge density and current are given by

$$
\begin{aligned}
& j_{t}=-\frac{\delta S_{D B I}}{\delta A_{t}}=T_{p} \lim _{z \rightarrow 0}\left[\sqrt{\frac{f(z) F(z)}{h(z)}} F_{z t}(z)\right]=T_{p} F_{z t}(0), \\
& j_{x}=-\frac{\delta S_{D B I}}{\delta A_{x}}=-T_{p} \lim _{z \rightarrow 0}\left[\sqrt{\frac{f(z) F(z)}{h(z)}} F_{z x}(z)\right]=-T_{p} F_{z x}(0) .
\end{aligned}
$$

Here we rescaled the gauge field as $A_{\mu} \rightarrow 2 \pi \alpha^{\prime} A_{\mu}$ so that the unit electric charge of the F-string is normalized to be one. This also replaces $\rho^{2} / R^{4}$ in (2.8) with $\left(2 \pi \alpha^{\prime}\right)^{2} \rho^{2} / R^{4}$ and thus in general AdS/CFT setups, the function $F(z)$ takes the form

$$
F(z)=\sqrt{a_{1}(\lambda)+a_{2}(\lambda) \cdot \rho^{2} z^{4}}
$$

where $a_{1}$ and $a_{2}$ are certain functions of the 't Hooft coupling $\lambda$, which depend on the details of setups. For the electric solution (2.7), we find that $\mu$ corresponds to the chemical potential and $\rho$ is proportional to the charge density via the relation $j_{t}=T_{p} \rho$. In this paper, we will always set $2 \pi \alpha^{\prime}=1$ just for a presentational simplification of our results.

\subsection{Calculating Holographic Conductivity}

By expanding the action around this solution (2.7) at the quadratic order about $A_{x}$, we obtain

$$
S_{D B I} \simeq T_{p} \int d t d x d y d z \sqrt{\frac{f(z) F(z)}{h(z)}}\left(\frac{F_{t x}^{2}}{f(z)}-h(z) F_{x z}^{2}\right) .
$$

The equation of motion for $A_{x}$ with the frequency $\omega$ i.e. $A_{x} \propto e^{-i \omega t}$ reads

$$
A_{x}^{\prime \prime}+\left(\frac{\partial_{z} \sqrt{h(z) f(z)}}{\sqrt{h(z) f(z)}}+g(z)\right) A_{x}^{\prime}+\frac{\omega^{2}}{h(z) f(z)} A_{x}=0,
$$

where we defined

$$
g(z)=\frac{\partial_{z} F(z)}{2 F(z)}
$$

It is useful to employ a new radial coordinate $w$ instead of $z$ defined by

$$
w(z)=\int_{0}^{z} \frac{d y}{\sqrt{h(y) f(y)}}
$$

The boundary $z=0$ corresponds to $w=0$. In all of our examples, $w$ runs from 0 to $\infty$ and the black brane horizon $z=z_{H}$ corresponds to $w=\infty$. 
At the same time, we rescale the gauge field $A_{x}$ into a new field $\Psi(w)$

$$
A_{x}(w)=F(z)^{-1 / 4} \cdot \Psi(w) .
$$

After this coordinate change $z=z(w)$ and rescaling of $A_{x}$, the Maxwell equation (2.13) takes the form of the Schrodinger equation

$$
-\Psi^{\prime \prime}(w)+V(w) \Psi(w)=\omega^{2} \Psi(w) .
$$

The effective potential is given by

$$
\begin{aligned}
V(w) & =\frac{1}{4} f(z) h(z) g(z)^{2}+\frac{1}{2} \partial_{w}[g(z) \sqrt{h(z) f(z)}] \\
& =\frac{1}{4} f(z) h(z) g(z)^{2}+\frac{1}{2} \sqrt{h(z) f(z)} \partial_{z}[g(z) \sqrt{h(z) f(z)}] .
\end{aligned}
$$

As pointed out in [47, we can relate the holographic calculation of conductivity to the following scattering problem in the Schrodinger problem. For this purpose, it is convenient to extend the range of the coordinate $w$ to $w<0$ assuming $V(w)=0$ there. Next we consider the following boundary value problem. First we impose the in-going boundary condition at the horizon $w=\infty$ [48]

$$
\Psi(w) \rightarrow T(\omega) \cdot e^{i \omega w} \quad(w \rightarrow \infty),
$$

where we implicitly use the fact that the potential $V(w)$ gets vanishing in this limit $w=\infty$ in all examples we are interested in. Then we solve (2.17) with this boundary condition toward the boundary $w=0$ to find the reflection coefficient $R(\omega)$ defined by

$$
\Psi(w) \rightarrow e^{i \omega w}+R(\omega) e^{-i \omega w} \quad(w \rightarrow 0) .
$$

In this way, we can uniquely obtain the reflection coefficient $R(\omega)$ and the transmission coefficient $T(\omega)$, which satisfy $|R(\omega)|^{2}+|T(\omega)|^{2}=1$. At the same time, this allows us to determine the holographic conductivity uniquely as follows

$$
\sigma(\omega)=\frac{j_{x}}{E_{x}}=\frac{T_{p} \cdot \partial_{w} A_{x}(0)}{i \omega A_{x}(0)}=T_{p} \cdot \frac{\partial_{w} \Psi(0)}{i \omega \Psi(0)}=T_{p} \cdot \frac{1-R(\omega)}{1+R(\omega)} .
$$

To evaluate the AC conductivity $\sigma(\omega)$ explicitly, we need to solve (2.17) numerically in general, as we will do in several examples later. However, it will be helpful to look at the simplest example where we find an exact expression of $\sigma(\omega)$. Consider the case where $g(z)$ vanishes and for example, this is realized if the charged density is vanishing $\rho=0$ and $\varphi(z)$ is constant. Even apart from our probe D-brane approximation, this corresponds to 
the case of the minimal Einstein-Maxwell theory with zero charge density. In such cases, the potential (2.18) clearly vanishes and thus there is no reflection $R(\omega)=0$. Therefore we find from (2.21) that the conductivity is independent of $\omega$

$$
\sigma(\omega)=T_{p}
$$

Even for general examples, since the effect of potential $V(w)$ gets smaller as we increase the energy $\omega^{2}$, we always find that in the high frequency limit $\omega / T \rightarrow \infty$

$$
\lim _{\omega \rightarrow \infty} \sigma(\omega)=T_{p}
$$

In the opposite case, where the potential $V(w)$ gives so large barrier that makes the tunneling effect impossible, we will find that the reflection coefficient $R(\omega)$ is purely a phase factor $e^{i \delta(\omega)}$ for small values of $\omega$. Then the real part of the conductivity $\operatorname{Re} \sigma(\omega)$ is vanishing. Such systems correspond to insulators because the DC conductivity clearly vanishes, while the others in our probe setup can be regarded as metals.

Another property which can be found analytically in generic cases is the sum rule. As we will explain in the appendix A, if there is no delta functional Drude peak, the AC conductivity in our setup always satisfies the simple sum rule (see also [12])

$$
\int_{0}^{\infty} d \omega\left(\operatorname{Re} \sigma(\omega)-T_{p}\right)=0 .
$$

\subsection{Conductivity}

Even for general systems, we can calculate the DC conductivity $\sigma_{D C}=\sigma(0)$ in an analytical way. This has been first calculated in [37] for several examples of probe D-branes in a different way. Here we derive the expression of $\sigma_{D C}$ as the $\omega \rightarrow 0$ limit of the AC conductivity $\sigma(\omega)$.

For this, it is useful to define a new function $X$ by

$$
X(w) \equiv \frac{\partial_{w} A_{x}(w)}{A_{x}(w)}=\frac{\partial_{w} \Psi}{\Psi}-\frac{1}{2} g(z) \sqrt{h(z) f(z)} .
$$

We often write equations using both the coordinate $z$ and $w$, as they are related by (2.15). The equation of motion (2.13) is rewritten to be

$$
\partial_{w} X=-g X \sqrt{h f}-X^{2}-\omega^{2}
$$

and the boundary condition at the horizon (2.19) is simply expressed as follows

$$
X(\infty)=i \omega
$$


Notice that (2.27) follows from (2.19) by assuming $\lim _{w \rightarrow \infty} g \sqrt{f h}=0$, which is essentially equivalent to the previous assumption $\lim _{w \rightarrow \infty} V(w)=0$.

The conductivity $\sigma(\omega)$ is found from the value of $X$ at the boundary $w=0$ via

$$
\sigma(\omega)=T_{p} \cdot \frac{X(0)}{i \omega}
$$

In the $\omega \rightarrow \infty$ limit, we find that the solution to (2.26) approaches to $X(w)=i \omega$ and we reproduce the behavior (2.23). Below we would like to find the behavior of $X(\omega)$ in the opposite limit $\omega \rightarrow 0$ to obtain the DC conductivity.

We can see from (2.26) and (2.27) that the real part $\operatorname{Re} \sigma(\omega)$ and the imaginary part $\operatorname{Im} \sigma(\omega)$ are even and odd function of $\omega$, respectively. They are also related by the Kramers-Kronig relation

$$
\operatorname{Im} \sigma(\omega)=\frac{1}{\pi} \cdot P \int_{-\infty}^{\infty} d \omega^{\prime} \frac{\operatorname{Re} \sigma\left(\omega^{\prime}\right)-T_{p}}{\omega-\omega^{\prime}}
$$

and thus if we know one of them, we can reproduce the other.

Let us first examine the behavior of the imaginary part in the $\omega \rightarrow 0$ limit. The nonvanishing $X$ in the $\omega \rightarrow 0$ limit means a pole at $\omega=0$ as follows from (2.28). This pole leads to a delta-functional peak in $\operatorname{Re} \sigma(\omega)$ as it can be directly confirmed by using (2.29). At finite temperature (or finite value of $z_{H}$ ), we can show that this pole does not appear in our probe systems because we can find the simple regular solution $X=0$ to (2.26) and (2.27) at $\omega=0$. This is natural as the phonon bath will introduce the dissipation and make the DC conductivity finite. On the other hand, the solution $X=0$ gets singular at zero temperature limit $z_{H} \rightarrow \infty$ unless

$$
\int_{0}^{\infty} d w g(w) \sqrt{h(z) f(z)}=\int_{0}^{z_{H}} d z g(z)<\infty .
$$

Next we turn to the real part $\operatorname{Re} \sigma(\omega)$ in the $\omega \rightarrow 0$ limit. Since $X$ is order $O(\omega)$, we can neglect $O\left(\omega^{2}\right)$ terms in $(2.26)$. This leads to

$$
X(w)=i \omega \cdot e^{\int_{w}^{\infty} d w g(w) \sqrt{h(z) f(z)}}=i \omega \sqrt{F\left(z_{H}\right)}
$$

where we employed (2.14) and our normalization $F(0)=1$. In this way, we obtain the simple expression of the DC conductivity $\sigma_{D C}$

$$
\begin{aligned}
\sigma_{D C} & \equiv \operatorname{Re} \sigma(0) \\
& =T_{p} \sqrt{F\left(z_{H}\right)}=T_{p} \sqrt{e^{-2 \varphi\left(z_{H}\right)}+\frac{\rho^{2}}{R^{4}} z_{H}^{4} e^{-\phi\left(z_{H}\right)}},
\end{aligned}
$$


which is finite and positive. If we remember that we set $2 \pi \alpha^{\prime}=1$, we find that the conductivity (2.32) is dimensionless as is typical in $2+1$ dimensional systems. For nondilatonic backgrounds, by using (2.11), the holographic DC conductivity takes the form

$$
\sigma_{D C}=\sqrt{b_{1}(\lambda)+b_{2}(\lambda) \frac{\rho^{2}}{T^{4}}},
$$

where $b_{1}(\lambda)$ and $b_{2}(\lambda)$ are certain model-depending dimensionless functions of the 't Hooft coupling $\lambda . \sigma_{D C}$ gets divergent at zero temperature for a non-vanishing charge density $\rho$. This is expected since there are no active gluons (or 'phonons') at zero temperature and there is no dissipation.

It might also be useful to compare (2.33) qualitatively with standard results in electron systems, though in our holographic case there is no clear quasi particle picture. The standard Drude formula is given by $\sigma_{D C}=\frac{n e^{2} \tau}{m}$, where $n, e, \tau$ and $m$ denote the electron density, charge, mean free time and mass. In our setup we can estimate $e=1, n \sim \rho$, $\tau \sim 1 / T$ and $m \sim T$. This indeed explains the behavior $\sigma_{D C} \sim \frac{\rho}{T^{2}}$ of (2.33) when $\rho$ is very large.

Finally, it is intriguing to explore possibilities of charged insulator systems in our probe brane setups. As is clear from (2.32), insulators are possible only if $e^{-2 \varphi\left(z_{H}\right)}=$ $\rho^{2} z_{H}^{4} e^{-\phi\left(z_{H}\right)}=0$. At zero temperature $z_{H} \rightarrow \infty$, this requires the infinitely large value of the dilaton in the IR limit of the supergravity background. In the dual gauge theory language, the IR theory gets infinitely strongly coupled. In this sense, we may think that this is analogous to Mott insulators, even though its gravity dual gets singular. In general, it is evident from (2.32) that the conductivity is reduced (or increases) if the dilaton gets large (or small) in the IR. We will present explicit results of AC conductivity in various systems from metals to insulators in the next section. Those readers who are mainly interested in holographic conductivities in disordered systems may jump to section $\mathbf{\text { Q }}$.

\section{AC Conductivity: From Metals to Insulators}

\subsection{Charged Probe Brane in AdS: Holographic Metal}

The most basic example of our probe D-brane setup is the charged probe D-brane in AdS Schwarzschild black branes. Though this has already been discussed in several earlier works e.g. [37, 40, 42], we first study this system from our viewpoint because this is the most basic example. Then we can assume that the metric (2.1) is given by the codimension 
one subspace of $A d S_{5}$ black brane

$$
f(z)=h(z)=1-\left(\frac{z}{z_{H}}\right)^{4},
$$

and that the dilaton and the compactified volume take constant values, which allows us to simply set $\phi(z)=\varphi(z)=0$. The functions $F(z)$ and $g(z)$ are

$$
F(z)=1+\frac{\rho^{2}}{R^{4}} z^{4}, \quad g(z)=\frac{2 \rho^{2} z^{3}}{R^{4}+\rho^{2} z^{4}} .
$$

The effective potential $V(w)$ defined by (2.18) is plotted in Fig.11. Here we chose and $R=z_{H}=1$. As is clear from its profile, there is a positive peak followed negative peak as $w$ gets larger. The former peak will reduce the conductivity at slightly high energy as it gives larger reflection, while the latter enhances the tunneling in the low energy. This expectation is indeed reflected in the plot of the (real part of) AC conductivity shown in Fig.2. We have set $T_{p}=1$. We can find a finite Drude peak in $\operatorname{Re} \sigma(\omega)$ and it eventually approaches to a constant $\operatorname{Re} \sigma(\omega)=1$ in the large $\omega$ limit, which is peculiar to the $2+1$ dimensional critical systems as we also explained in (2.23). In this probe system, the gluon sector plays a role of phonons and this leads to the dissipations. Thus we do not get the delta-functional Drude peak but get the smooth finite Drude peak at non-zero temperature. In the zero temperature limit, it changes into a delta-functional peak as we can see from the fact that the value of $F\left(z_{H}\right)$, which is proportional to the DC conductivity (2.32), gets divergent in the limit $z_{H} \rightarrow \infty$.

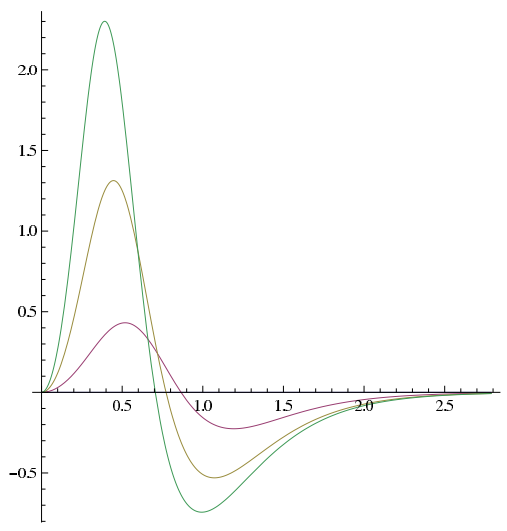

Figure 1: The effective potential $V(w)$ for the charge probe D-brane in AdS. It corresponds to $\rho=0$ (blue), 1(red), 2(yellow), 3(green). 

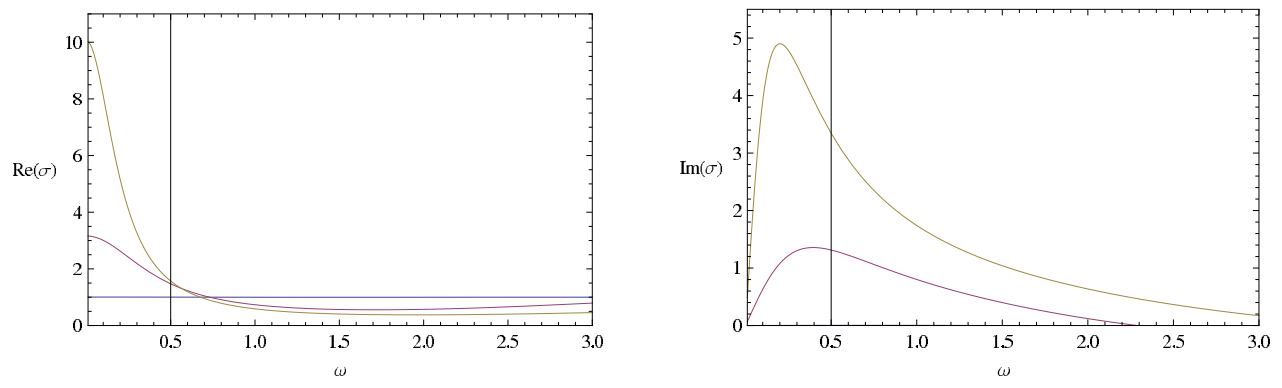

Figure 2: The real and imaginary part of conductivity from charged probe brane in AdS. We took $\rho=0.1$ (blue), 3 (red), 10 (yellow).

\subsection{Probe Brane vs. Bulk Approach in Holographic Metals and Superconductors}

Before we go on, it is useful to remind us of holographic calculations of conductivity in bulk gravity duals such as the ones in Einstein-Maxwell theory without introducing any probe D-branes. We would like to make their difference clearer in spite of their similar looking.

First, it is clear that a charged AdS black brane in Einstein-Maxwell theory is dual to a metallic system. In this example, if we plot the $\mathrm{AC}$ conductivity, we find a deltafunctional Drude peak even at non-zero temperature. This unusual behavior is because the system does not coupled to any other sectors as opposed to the previous probe Dbrane example and thus there are no external objects analogous to phonons. This fact is manifest in the equation of motion of the gauge field, which is obtained after we combine the Einstein equation with Maxwell equation as calculated in [5]

$$
A_{x}^{\prime \prime}+\left(\frac{f^{\prime}(z)}{f(z)}\right) A_{x}^{\prime}+\left(\frac{\omega^{2}}{f(z)^{2}}-\frac{Q^{2} z^{2}}{f(z)}\right) A_{x}=0
$$

where we set $f(z)=1-\left(1+Q^{2}\right) z^{3}+Q^{2} z^{4}$. The charge of the AdS black brane is denoted by $Q$. The important point is that the effect of non-zero charge enters via the coefficient in front of $A_{x}$. This makes a sharp contrast with the probe D-brane case where the charge effect appears in the coefficient of $A_{x}^{\prime}$ term as in (2.13). In this bulk gravity dual case, we have instead of (2.26)

$$
\partial_{w} X=-X^{2}-\omega^{2}-Q^{2} z^{2} f(z)
$$

Thus $X$ gets non-vanishing at the boundary $z=0$, leading to a pole of $\operatorname{Im} \sigma(\omega)$ at $\omega=0$, leading to a delta functional Drude peak via the Kramers-Kronig relation. The 
Schrodinger potential for the equation (3.36) is given by

$$
V(w)=Q^{2} z^{2} f(z)
$$

As the $Q$ increases, the potential barrier grows. Therefore the reflection increases and thus $\operatorname{Re} \sigma(\omega)$ is reduced for non-zero small $\omega$. The explicit form of the potential $V(w)$ is plotted in Fig. 3 .

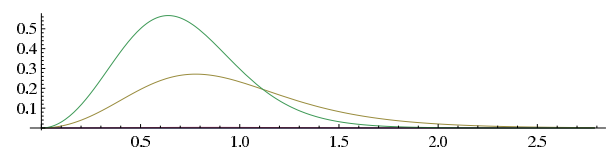

Figure 3: The effective potential $V(w)$ for the charge AdS black brane for various charges $Q=0,0.1,1, \sqrt{3}$.

One may think that this delta functional Drude peak is very similar to that of holographic superconductors [12, 5, 6] as superconductors are characterized by an infinitely large DC conductivity. Indeed, in the bulk gravity dual, the calculation of conductivity for holographic superconductors goes very similarly. Basically, we just need to replace $Q^{2}$ with $Q^{2}+|\Phi|^{2}$ in (3.36) and (3.38), where $\Phi$ is a complex scalar field whose condense triggers the superconductor phase transition. Therefore the effect of $\Phi$ condensation is very similar to that of non-zero charge $Q$.

However, once we promote them to probe D-brane setups [49, we will notice the crucial difference between holographic superconductors and charged AdS black branes. At finite temperature, the former still has a delta functional Drude peak as the effect of scalar field always come in $A_{x}$ term, while the latter leads to a finite Drude peak as the effect only appears in the $A_{x}^{\prime}$ term. In this way, there is a sharp distinction between the superconductors and metals in the probe D-brane approach.

\subsection{Holographic Metals with Pseudo Gaps}

It is very interesting to understand what will happen if we modify the supergravity background of a charged probe brane. One important class of modifications is to assume a non-trivial profile of the dilaton $\phi$. Another will be to consider different metrics than the AdS, such as the Lifshitz backgrounds [14]. Since there has already been considerable works in the latter, we will focus on the former below.

\footnotetext{
${ }^{5}$ For the analysis of holographic conductivity in Lifshitz backgrounds refer to e.g. [15, 41, 43, 44.
} 
Basically, there are two possibilities of the behavior of dilaton at zero temperature: the dilaton gets smaller or larger in the IR limit $w \rightarrow \infty$. In this subsection we will study the former, while the latter will be discussed in the next subsection.

Here we will study the type IIB supergravity solution found in [47] as an example of such a dilatonic background. The metric of this black brane solution reads in the Einstein frame

$$
\begin{aligned}
& d s^{2}=R^{2}\left[z^{-2}\left(-F(r) d t^{2}+d x^{2}+d y^{2}\right)+z^{-4 / 3} d \chi^{2}+\frac{d z^{2}}{z^{2} F(z)}\right]+\tilde{R}^{2} d s_{X_{5}}^{2}, \\
& F(r)=1-M \cdot z^{11 / 3}
\end{aligned}
$$

where $X_{5}$ is an arbitrary Einstein manifolds. $M$ is a mass parameter of the black brane which is proportional to the temperature. On the other hand, the dilaton behaves $e^{\phi(z)} \propto z^{-2 / 3}$ and goes to zero in the IR limit $z \rightarrow \infty$ as we promised. This solution is supported by RR 5-form and 1-form fluxes and can be regarded as a gravity dual of (non-supersymmetric) D3-D7 system. The holographic dual gauge theory lives in the $3+1$ dimension spanned by $(t, x, y, \chi)$ and from this viewpoint, the $\chi$ coordinate has an anisotropic scaling property. In this sense, this background (3.39) can be regarded as a gravity dual of space-like Lifshitz fixed point. However, for our purpose, a probe D3-brane is assumed to extend in $(t, x, y, r)$ direction in $X_{5}$ and therefore the probe brane world-volume looks like a AdS space or its black brane, rather than a Lifshitz background. Notice that in this setup we have $\phi(z)=\varphi(z)$ because the radius of $S^{5}$ denoted by $\tilde{R}$ does not depend on $z$.

As shown in [47, we can construct an interpolating solution which starts from the conventional $\mathrm{AdS}_{5} \times \mathrm{S}^{5}$ solution at the boundary $z=0$ and approaches the dilatonic solution (3.39) in the IR limit $z \rightarrow \infty$. Since this solution is highly complicated, below we will replace it with the simple profile of the dilaton

$$
e^{\phi(z)}=e^{\varphi(z)}=\frac{1}{\left(1+z^{2}\right)^{1 / 3}},
$$

with the metric (3.39) unchanged. Since the relevant physics is essentially hidden in the scaling metric (3.39), this approximation is enough for our purpose.

First we start with calculating the holographic conductivity at zero temperature $M=$ 0 . The effective potential $V(z)$ behaves like

$$
V(z) \rightarrow \frac{1}{3}(z \rightarrow 0), \quad V(z) \rightarrow \frac{7}{36 z^{2}} \quad(z \rightarrow \infty),
$$

and its explicit form of the potential is plotted in Fig. 1. After solving the Schrodinger problem, the resulting conductivity is shown in Fig.5. There is a delta-functional Drude 
peak in $\operatorname{Re} \sigma(\omega)$ hidden at $\omega=0$ as is clear from the behavior of $\operatorname{Im} \sigma(\omega)$ at $\omega=0$ and also from the fact that $F\left(z_{H}\right) \rightarrow \infty$ in the limit $z_{H} \rightarrow \infty$. Also we can confirm for small $\omega$

$$
\operatorname{Re} \sigma(\omega) \propto \omega^{1 / 3}(+\delta(\omega) \text { term }) .
$$

The reason why this vanishes in the limit $\omega \rightarrow 0$ is that the potential $V(z) \sim z^{-2}$ for large $z$ (3.41) does not allow tunneling at zero energyf. In this sense, the system dual to the D3-brane in our dilatonic background behaves like a metal with a pseudo gap.

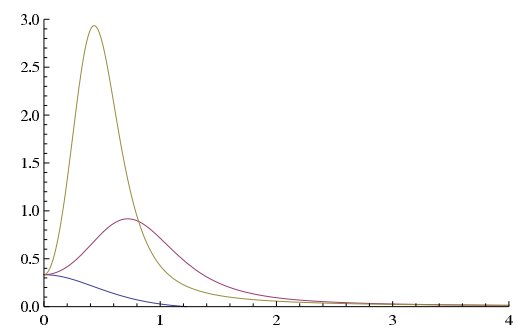

Figure 4: The effective potential $V(w)$ of charged probe brane in the dilatonic background (3.39) at zero temperature for $\rho=0$ (blue), $\rho=1$ (red) and $\rho=3$ (yellow).
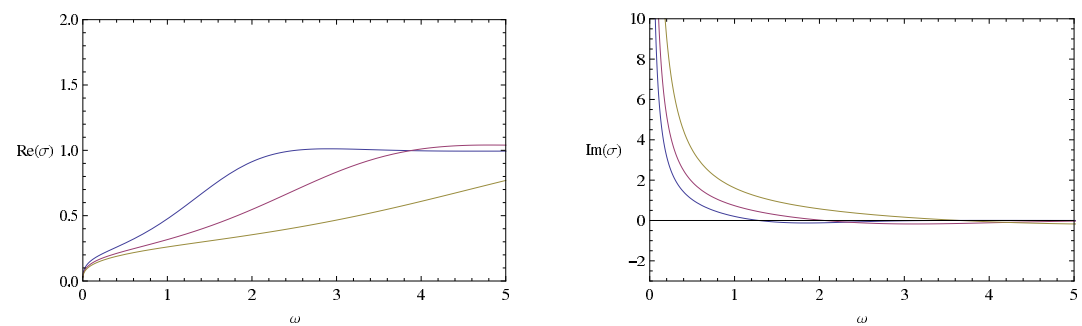

Figure 5: The plot of real and imaginary part of the conductivity for the probe brane in the dilatonic background (3.39) at zero temperature for $\rho=1$ (blue), $\rho=3$ (red) and $\rho=10$ (yellow).

At finite temperature, corresponding to positive values of $M$ in (3.39), the effective potential behaves like

$$
V(0)=\frac{1}{3}, \quad V(w) \rightarrow O\left(e^{-w}\right) \quad(w \rightarrow \infty) .
$$

${ }^{6}$ Such a power behavior of the AC conductivity has been already known in various calculations of holographic conductivity [15, 41, 43] with or without probe approximations. Our example here can be regarded as a particular example of these classes and what we showed here is that we can embed this into type IIB string theory. 
The explicit form of the potential is plotted in Fig.6. The AC conductivity at finite temperature is shown in Fig.77.
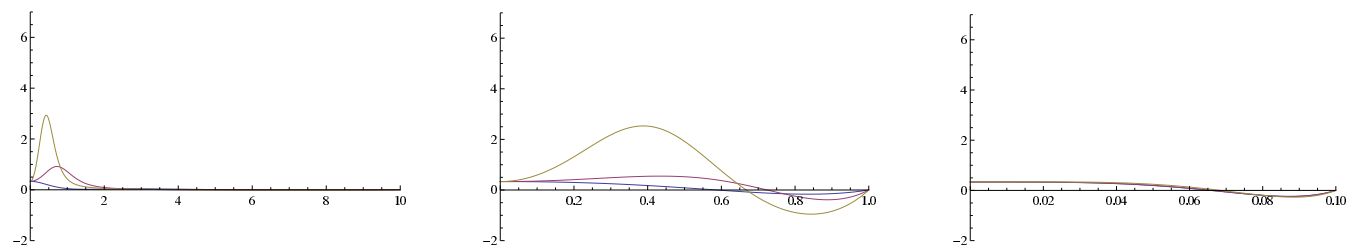

Figure 6: The effective potential $V(z)$ for the finite temperature dilatonic solution (3.39). The temperature is given by $M=0.1$ (left), $M=1$ (middle), $M=10$ (right). The charge density is $\rho=0.1$ (blue), $\rho=1$ (red) and $\rho=3$ (yellow).
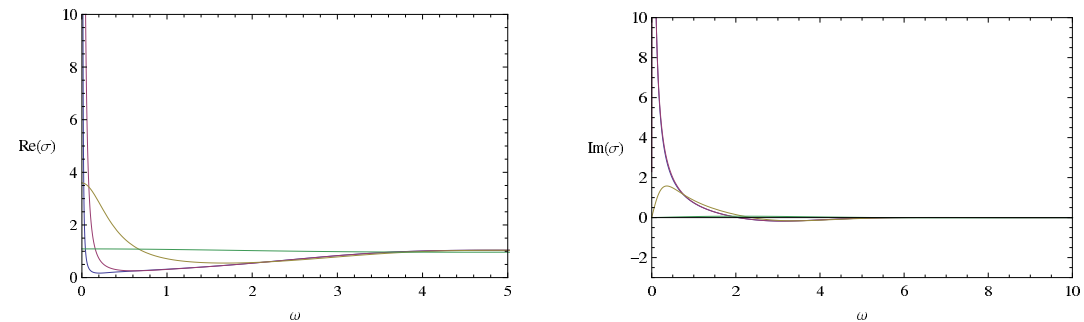

Figure 7: The plot of real and imaginary part of the conductivity for the probe brane in the finite temperature dilatonic solution (3.39). $(M=0.1$ (blue), $M=0.3$ (red), $M=1$ (yellow), $M=3$ (green). All have the charge density $\rho=3$.

As is clear from this potential, as the temperature increases, the sharp potential wall at small $z$ spreads and a negative potential region develops in the near horizon region $z>>1$. Accordingly, the delta functional Drude peak at zero temperature becomes broad and the pseudo gap gradually disappears as the temperature increases. The high temperature behavior is similar to the AdS BH (i.e. Fig.11).

\subsection{Towards Holographic Mott Insulators}

As final examples, we would like to consider possibilities of constructing gravity duals of insulators at non-zero charge density]. For this, we need to realize the vanishing DC

\footnotetext{
${ }^{7}$ There are earilar works on holographic insulators. At vanishing charge density, one clear example of holographic insulators is the AdS soliton [50]. In the charged black hole background, it has been shown that interactions can make fermions gapped in [52]. Also the system whose AC conductivity shows the
} 
conductivity at zero temperature. This is possible in our probe D-brane setup only if the dilaton gets infinitely large in the IR limit $z \rightarrow \infty$ as we already mentioned. Even though in such an example we need to worry about the singular behavior due to the strongly coupled limit, we will ignore this issue and proceed to the calculations of conductivity assuming that an appropriate string duality will make the background non-singular.

We will focus on the zero temperature limit, setting $h(z)=f(z)=1$ and analyze the following three different profiles $(i)-($ iii $)$ of the dilaton

$$
\begin{aligned}
& \text { (i) Pseudo-gap insulator : } e^{\phi(z)}=e^{\varphi(z)}=1+z^{8} \text {, } \\
& \text { (ii) Soft-gap insulator : } e^{\phi(z)}=e^{\varphi(z)}=e^{\left(1+z^{4}\right)^{\frac{1}{8}}} \text {, } \\
& \text { (iii) Hard-gap insulator : } e^{\phi(z)}=e^{\varphi(z)}=e^{\left(1+z^{4}\right)^{\frac{1}{4}}} \text {. }
\end{aligned}
$$

We leave explicit constructions of corresponding dilatonic backgrounds as supergravity solutions for a future problem. The effective potential for each background behaves as follows:

$$
\begin{array}{lll}
\text { (i) } V(w) \sim O\left(w^{2}\right) \quad(w \rightarrow 0), & V(w) \sim \frac{2}{w^{2}}(w \rightarrow \infty), \\
\text { (ii) } V(w) \sim O\left(w^{2}\right) \quad(w \rightarrow 0), & V(w) \sim \frac{1}{16 w} \quad(w \rightarrow \infty), \\
\text { (iii) } V(w) \sim O\left(w^{2}\right)(w \rightarrow 0), & V(w) \sim \frac{1}{16}(w \rightarrow \infty) .
\end{array}
$$

The explicit forms of these potentials are plotted in Fig.8. The holographic conductivity for each insulators are presented in Fig.9, Fig.10 and Fig.11, respectively.

In summary, we obtain the following behaviors of the $\mathrm{AC}$ conductivity at low frequency:

(i) Pseudo-gap insulator: $\operatorname{Re} \sigma(\omega) \rightarrow \omega^{2} \quad(\omega \rightarrow 0)$,

(ii) Soft-gap insulator: $\operatorname{Re} \sigma(\omega) \rightarrow e^{-\frac{0.048}{\omega}} \quad(\omega \rightarrow 0)$,

(iii) Hard-gap insulator : $\operatorname{Re} \sigma(\omega)=0 \quad\left(0 \leq \omega<\frac{1}{4}\right)$.

Notice that in all of these examples there are no delta functional Drude peaks and therefore they correspond to various kinds of insulators.

power law behavior (we called this a metal with a pseudo gap) can be regarded as a Mott insulator if we can get rid of the delta functional Drude peak 43, 51. 


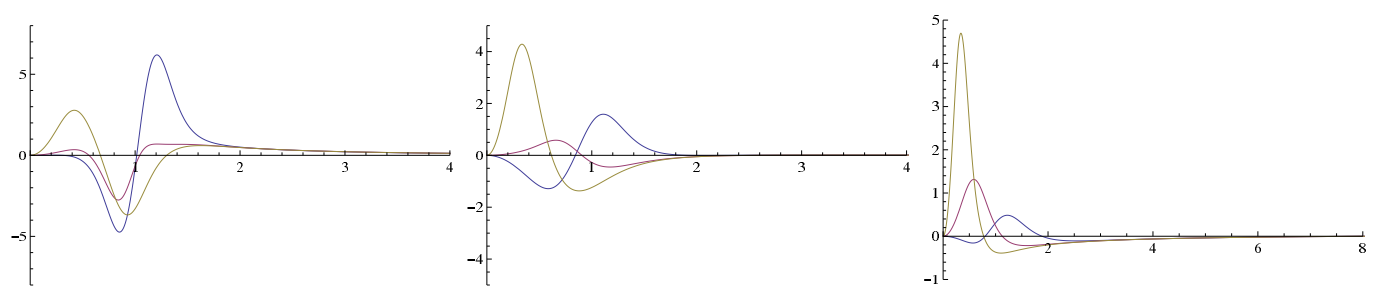

Figure 8: The effective potentials $V(w)$ for the insulator models (3.44), (3.45) and (3.46) each corresponds to in the left, middle and right graph, respectively. Each colored curve represents different charge densities: $\rho=0.3$ (blue), $\rho=1$ (red) and $\rho=3$ (yellow).
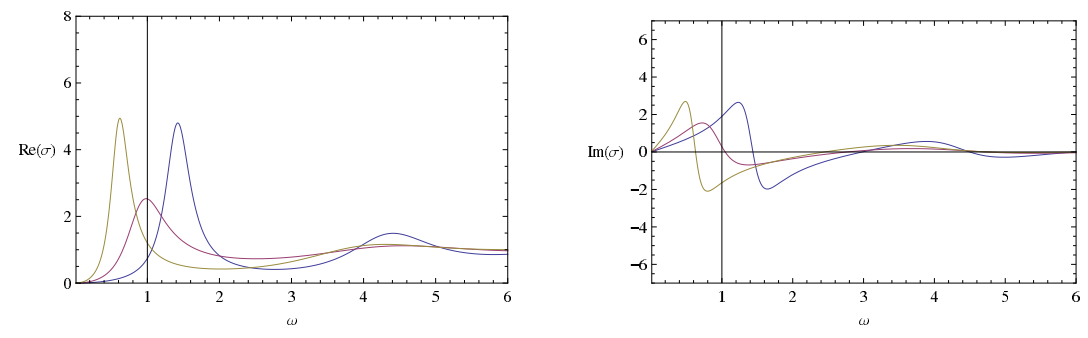

Figure 9: The plot of real and imaginary part of the conductivity for the insulator model (i) at $\rho=0.3$ (blue), $\rho=1$ (red) and $\rho=3$ (yellow).
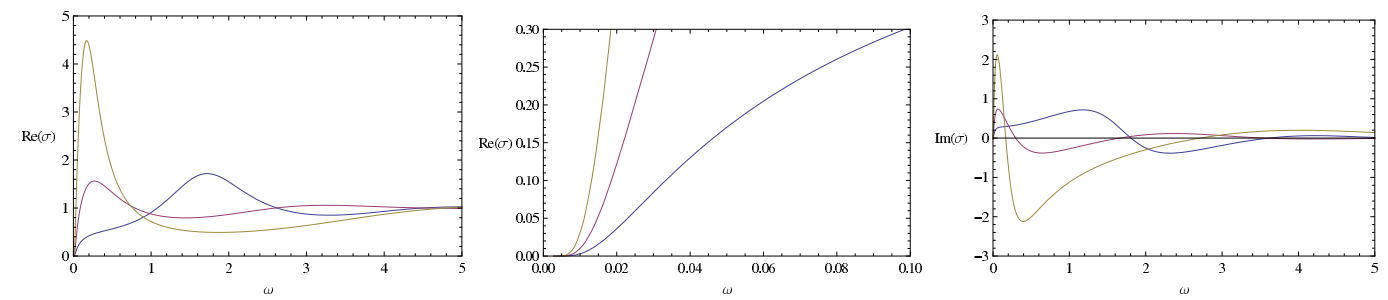

Figure 10: The plot of real (the first and second graph) and imaginary part (the last one) of the conductivity for the insulator model (ii) at $\rho=0.3$ (blue), $\rho=1$ (red) and $\rho=3$ (yellow). 

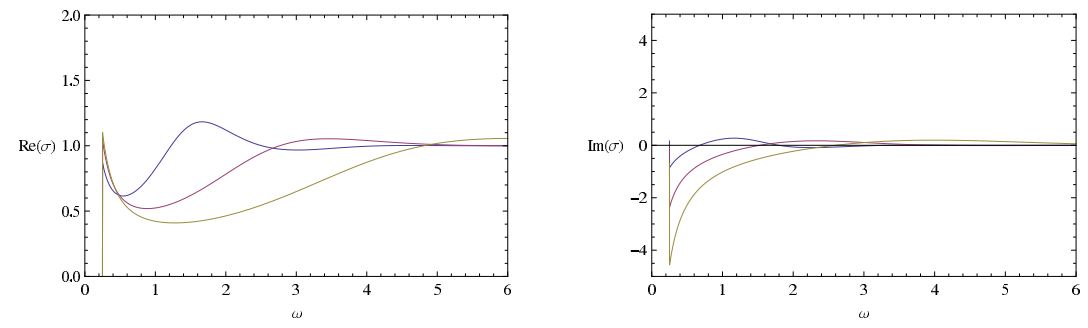

Figure 11: The plot of real and imaginary part of the conductivity for the insulator model (iii) at $\rho=0.3$ (blue), $\rho=1$ (red) and $\rho=3$ (yellow). 


\section{Holographic Conductivity in Disordered Systems}

So far we have analyzed the holographic conductivity in various probe D-brane setups. In some sense, they are analogous to systems with electrons interacting with phonons and owing to it the DC conductivity gets finite. However, in realistic materials, they usually include static impurities and they cause the reduction of conductivity. The main purpose of this paper to incorporate the effect of impurities in the holographic frame work and this section will be devoted to this problem. We treat impurities as quenched disorder, which means time-independent randomness, as is standard in condensed matter physics 23, 24] (see also e.g. reviews [25, 26, 27]).

\subsection{Origins of Randomness in Probe Brane Setup}

We can consider several origins of randomness in our probe D-brane setups. They are largely divided into two classes. One of them is the random fluctuations of the background closed string fields (or supergravity fields) such as the dilaton $\phi(x, y, z)$ or the metric

$g_{\mu \nu}(x, y, z)$. For example, if we regard the impurities as D-branes or other solitonic objects in string theory localized in $x$ and $y$ direction and distributed randomly (similar to the idea in [32), then its backreaction clearly induces such random fluctuations of closed string fields. This class is regarded as providing charge neutral impurities. The other is those arising from the background open string fields, in particular the gauge potential $A_{t}$. This is regarded as a charged impurity potential. Also if the closed string background fluctuates, then the solution (2.7) with non-zero charge density itself will be modified such that $A_{t}$ takes random values. Since we are interested in how disorder affects low energy physics, we can assume that the randomness disappears near the boundary $z=0$. We will analyze these various disordered effects separately. In Sections 4.6 and 4.7, we will study random fluctuations of charge density and dilaton field, respectively.

An important issue when we consider the metric fluctuations is that the horizon, which exists uniformly in a finite temperature gravity dual, can locally disappear depending on the position of $(x, y)$ in a random way. This means that the spacetime can locally change from a AdS black brane to an AdS soliton. An AdS soliton denotes here an asymptotically AdS spaces whose IR regions are capped off. A famous example is the one which can be obtained from a double Wick rotation of AdS black brane and this has been important in the context of confinement/deconfinement phase transition [53, 54. The AdS soliton itself behaves like a holographic insulator [50]. This fluctuation, which makes a 'hole' on the horizon, turns out to be very important to understand a transition from a metal into an insulator in our holographic setup as we will see in section 4.8. This is because such a 
hole is interpreted as an impurity which leads to a repulsive interaction due to the mass gap dual to the AdS soliton geometry. This effect can be approximately incorporated as the change of boundary condition at the horizon as we will explain later.

Below we will analyze how the calculation of conductivity is changed due to the randomness. In order to obtain analytically exact results, we will first assume that the random background only depends on the coordinate $x$ but not on $y$, which corresponds to codimension one (or string shape) impurities. Finally, we will discuss the holographic conductivity when the randomness depends both on $x$ and $y$ in section 4.9 .

\subsection{Definition of Conductivity in Disordered Systems}

Before we proceed to the holographic calculation, it is important to make clear the definition of conductivity in disordered systems. Below we will follow the argument in [55]. The presence of impurities breaks the translational invariance and thus we encounter a bilocal conductivity $\sigma_{i j}\left(x, x^{\prime}, \omega\right)$ defined by the linear response between the current and electric field

$$
j_{i}(x, \omega)=\int d x^{\prime} \sigma_{i j}\left(x, x^{\prime}, \omega\right) E_{j}\left(x^{\prime}, \omega\right),
$$

where $i, j$ run over the space coordinates of a given system. Since in this paper we are only interested in the case $i=j=x$ and so we simply write $\sigma_{x x}$ as $\sigma$.

If we perform the random average denoted by $\langle\ldots\rangle$, the translational invariance is recovered and we can write it as follows:

$$
\left\langle\sigma\left(x, x^{\prime}, \omega\right)\right\rangle=\sigma\left(x-x^{\prime}, \omega\right)
$$

Then we can define the AC conductivity in the disordered system as follows

$$
\sigma(\omega) \equiv \sigma(k=0, \omega)=\int d x \sigma(x, \omega)
$$

Now we would like to consider a simpler but equivalent definition of the conductivity in the disordered systems. We fix the external electric field as $E_{x}\left(x^{\prime}, \omega\right)=E_{x}(\omega) e^{i k x^{\prime}}$ so that it has a definite wave number $k$. In this case $j_{x}$ includes various wave numbers in general. However, after the disorder average we will get

$$
\left\langle j_{x}(x, \omega)\right\rangle=\sigma(k, \omega) E_{x}(\omega) e^{i k x}
$$

8 In this paper we employ the normalization of Fourier transformation such that $A(x)=$ $\frac{1}{2 \pi} \int d k e^{i k x} A(k)$ and $A(k)=\int d x e^{-i k x} A(x)$ for any function $A$. 
Thus we obtain for the wave number $k$ electric field

$$
\frac{\left\langle j_{x}(x, \omega)\right\rangle}{E_{x}(x, \omega)}=\sigma(k, \omega) .
$$

By taking $k \rightarrow 0$, we eventually find

$$
\sigma(\omega)=\frac{\left\langle j_{x}(x, \omega)\right\rangle}{E_{x}(\omega)},
$$

where notice that the random average $\left\langle j_{x}(x, \omega)\right\rangle$ actually does not depend on $x$.

\subsection{Analysis of Equations of Motion}

First we derive the equation of motion for the gauge field in DBI action for the general probe D-brane. In particular, we consider a generalized black brane background where the scalar fields $\phi$ and $\varphi$ depend on both $x$ and $z$ and the metric is given by (2.1) with $h(z)=f(z)$. We define a function $F(z, x)$ by generalizing the previous one (2.8) by (below we suppress the $R$ dependence by setting $R=1$ just for simplicity)

$$
\sqrt{F(z, x)} \equiv \frac{e^{-\varphi(z, x)}}{\sqrt{1-e^{-\phi(z, x)} z^{4} F_{t z}^{2}-\frac{z^{4} e^{-\phi(z, x)}}{f(z)} F_{t x}^{2}+z^{4} f(z) e^{-\phi(z, x)} F_{z x}^{2}}} .
$$

The equations of motion of gauge fields from the DBI action (2.6) are given as follows for the $t, x$ and $z$ component:

$$
\begin{aligned}
& \partial_{z}\left(\sqrt{F(z, x)} \partial_{z} A_{t}\right)+\partial_{x}\left(f(z)^{-1} \sqrt{F(z, x)}\left(\partial_{x} A_{t}-\partial_{t} A_{x}\right)\right)=Q(x, z), \\
& \partial_{t}\left(f(z)^{-1} \sqrt{F(z, x)}\left(\partial_{t} A_{x}-\partial_{x} A_{t}\right)\right)-\partial_{z}\left(f(z) \sqrt{F(z, x)} \partial_{z} A_{x}\right)=0, \\
& \partial_{t}\left(\sqrt{F(z, x)} \partial_{z} A_{t}\right)-\partial_{x}\left(f(z) \sqrt{F(z, x)} \partial_{z} A_{x}\right)=0,
\end{aligned}
$$

where $Q(x, z)$ is the random charged source mentioned in the previous subsection.

It is clear that the static solution with a charge can be constructed in the form (remember also the gauge condition $A_{z}=0$ )

$$
A_{t}=\alpha(x, z), \quad A_{x}=0 .
$$

The profile of $\alpha(x, z)$ is found by solving

$$
\partial_{z}\left(\sqrt{F} \partial_{z} \alpha\right)+\partial_{x}\left(f^{-1} \sqrt{F} \partial_{x} \alpha\right)=Q(x, z),
$$


with the standard boundary condition at the horizon $\alpha\left(z_{H}\right)=0$. As we mentioned, it is sensible to assume that there is no randomness at the boundary $z=0$ and thus we can always set

$$
F(0, x)=1
$$

Next we consider perturbations $A_{t}$ and $A_{x}$ around this solution to calculate the conductivity. The detailed form of the function $\alpha$ is not important in the following arguments. After we perform the Fourier transformation of $t$ into $\omega$, the perturbative equations of motion read

$$
\begin{aligned}
& f \partial_{z}\left(f \sqrt{F} \partial_{z} A_{x}\right)+\left(\sqrt{F}+\frac{K}{f}\left(\partial_{x} \alpha\right)^{2}\right)\left(\omega^{2} A_{x}-i \omega \partial_{x} A_{t}\right)-i \omega K\left(\partial_{x} \alpha\right)\left(\partial_{z} \alpha\right) \partial_{z} A_{t}=0 \\
& \partial_{x}\left(f \sqrt{F} \partial_{z} A_{x}\right)+i \omega\left(\sqrt{F}+K\left(\partial_{z} \alpha\right)^{2}\right) \partial_{z} A_{t}+\frac{K}{f}\left(\partial_{x} \alpha\right)\left(\partial_{z} \alpha\right)\left(-\omega^{2} A_{x}+i \omega \partial_{x} A_{t}\right)=0 .
\end{aligned}
$$

We defined the function $K(z, x)$ by

$$
K(z, x)=\frac{z^{4} e^{-\varphi-\phi}}{\left(1-e^{-\phi} z^{4} F_{t z}^{2}-\frac{z^{4} e^{-\phi}}{f} F_{t x}^{2}+z^{4} f e^{-\phi} F_{z x}^{2}\right)^{3 / 2}} .
$$

$F(z, x)$ and $K(z, x)$ in (4.61) and (4.62) are evaluated in the gauge field background (4.58). Note that they come from $x$ and $z$ component of the equations of motion of gauge fields and we did not write explicitly that from $t$ component as it is not independent from (4.61) and (4.62).

As we notice by plugging (4.58) in (4.56) or (4.63), in order to have a smooth background, the function $\alpha(z, x)$ should behave near the horizon as

$$
\alpha(z, x)=\beta(x)\left(z_{H}-z\right)+O\left(\left(z_{H}-z\right)^{2}\right) \quad\left(z \rightarrow z_{H}\right)
$$

where the function $\beta(x)$ is a random function, which is determined by an explicit random background we choose.

\subsection{Boundary Conditions}

Since the equations of motion are generally second order, we need one boundary condition for each field. In black brane backgrounds, we impose this at the horizon. For the gauge potential $A_{t}$ we require

$$
A_{t}\left(z_{H}, x\right)=0 \text {, }
$$


as usual. Note that this corresponds to a fixing of the residual gauge transformation $\delta A_{\mu}=\partial_{\mu} \chi(t, x)$. On the other hand, for the gauge field $A_{x}$, we impose the in-going boundary condition at the horizon. By using the condition (4.65) and equations (4.61) and (4.62), we find that $A_{x}$ satisfies in the near horizon limit $w \rightarrow \infty$

$\frac{1}{\sqrt{F}} \partial_{w}\left(\sqrt{F} \partial_{w} A_{x}\right)+\omega^{2}\left(1+\frac{K\left(\partial_{x} \alpha\right)^{2}}{f\left(\sqrt{F}+K\left(\partial_{z} \alpha\right)^{2}\right)}\right) A_{x}+\frac{K\left(\partial_{x} \alpha\right)\left(\partial_{z} \alpha\right)}{\sqrt{F}\left(\sqrt{F}+K\left(\partial_{z} \alpha\right)^{2}\right)} \partial_{x}\left(\sqrt{F} \partial_{w} A_{x}\right)=0$

Moreover by using the condition (4.64), we can neglect terms proportional to $\partial_{x} \alpha$ in (4.66) in the near horizon. Therefore $A_{x}$ satisfied the same equation as (2.13) and ingoing boundary condition is again given by (2.19). By generalizing the function (2.25), define

$$
X(w, x)=\frac{\partial_{w} A_{x}(w, x)}{A_{x}(w, x)},
$$

and then we have the integral expression

$$
A_{x}(w, x)=A_{x}(0, x) \cdot e^{\int_{0}^{w} d w X(w, x)} .
$$

Now the in-going boundary condition at the horizon is simply written as

$$
X(\infty, x)=i \omega .
$$

\subsection{Calculation of DC Conductivity}

After the previous preparations, we are now in a position to holographically calculate the DC conductivity in quenched disordered systems following the definition (4.55). In the Fourier basis, we normalize the external electric field $E_{x}=F_{t x}$ such that it is given by $-i \omega$ at the boundary

$$
E_{x}(0, x)=-i \omega A_{x}(0, x)-\partial_{x} A_{t}(0, x)=-i \omega .
$$

All we need to solve are (4.61) and (4.62) under the boundary conditions (4.65), (4.69) and (4.70). The AC conductivity is obtained from

$$
\sigma(\omega)=T_{p}\left\langle\frac{X(0, x)}{i \omega} \cdot A_{x}(0, x)\right\rangle
$$

where again $\langle\cdots\rangle$ denotes the random average.

For generic $\omega$, we have to resort to a rather complicated numerical analysis to find $\sigma(\omega)$. However, if we are interested in DC conductivity $\sigma(0)$ for codimension one impurities, we can actually obtain an analytical result and this is one of the main results in this paper. 
Concentrating on the DC limit $\omega \rightarrow 0$, we can expand fields in a series of $\omega$ as follows

$$
\begin{aligned}
& X(w, x)=i \omega \cdot a(w, x)+O\left(\omega^{2}\right), \\
& A_{t}(0, x)=i \omega \cdot p(x)+O\left(\omega^{2}\right), \\
& A_{x}(0, x)=1-\partial_{x} p(x)+O(\omega),
\end{aligned}
$$

where we imposed (4.70). $a(w, x)$ and $p(x)$ are functions which will be fixed by the equations of motion. I The DC conductivity is now given, by taking the limit $\omega \rightarrow 0$ of (4.71), by

$$
\sigma(0)=T_{p}\left\langle a(0, x)\left(1-\partial_{x} p(x)\right)\right\rangle .
$$

The boundary condition (4.88) is translated into

$$
a(\infty, x)=1 .
$$

In the DC limit (4.72), the equations of motion (4.61) and (4.62) are simply reduced to

$$
\partial_{w}\left(\sqrt{F} \partial_{w} A_{x}\right)=\partial_{x}\left(\sqrt{F} \partial_{w} A_{x}\right)=0 .
$$

Employing (4.67) and (4.68) in the DC limit, we find that the combination

$$
\sigma_{D C} \equiv T_{p} \sqrt{F(z, x)} \cdot\left(1-\partial_{x} p(x)\right) \cdot a(w, x),
$$

is a constant $\square$ which does not depend on $x$ and $z$. Indeed, this quantity $\sigma_{D C}$ coincides with the DC conductivity we want to compute as is clear from (4.60) and (4.73). By setting $z=z_{H}$ with the condition (4.74), we have the expression

$$
\sigma_{D C}=T_{p} \sqrt{F\left(z_{H}, x\right)} \cdot\left(1-\partial_{x} p(x)\right) .
$$

To fix the value of the constant $\sigma_{D C}$, we need to look at the behavior of the charge density $\rho$. As is clear from the definition (2.9), it is perturbed by the linear fluctuations which we exert in order to calculate the linear response. Thus this perturbation of the charge density depends on $p(x)$. Therefore, this physical consideration leads to the constraint require

$$
\lim _{L \rightarrow \infty} \frac{1}{L} \int_{0}^{L} \partial_{x} p(x)=0
$$

\footnotetext{
${ }^{9}$ Note that $p(x)$ cannot be eliminated by gauge transformations because we impose the condition (4.65).

${ }^{10}$ One may wonder why the current $j_{x}$ and conductivity do not depend on $x$ in spite of the $x$ dependent randomness. This is explained by the current conservation $\frac{\partial j_{x}}{\partial x}=-\frac{\partial \rho}{\partial t}=O\left(\omega^{2}\right)$ if we remember $\rho \sim$ $\partial_{z} A_{t} \sim O(\omega)$. This simple property is only true for our codimension one impurities.
} 
where $L$ is the length in the $x$ direction and should be taken to be infinity. This relation says that the charge density should not change a lot in the presence of the disorder and is clear if we impose a periodic boundary condition $p(x+L)=p(x)$ or a damping condition at the boundaries $x=0$ and $x=L$. This requirement (4.78) allows us to obtain the final expression of the DC conductivity after taking the random average:

$$
\sigma_{D C}=T_{p}\left\langle\frac{1}{\frac{1}{L} \int_{0}^{L} d x \frac{1}{\sqrt{F\left(z_{H}, x\right)}}}\right\rangle .
$$

Notice also that the formula 4.79$)$ is reduced to the previous result (2.32) if we turn off the randomness.

Since the function $\frac{1}{x}$ is convex, we can easily see

$$
\sigma_{D C} \leq T_{p}\left\langle\frac{1}{L} \int_{0}^{L} d x \sqrt{F\left(z_{H}, x\right)}\right\rangle .
$$

Notice that the right-hand side of the above inequality is a naive average of conductivity based on the result (2.32) without disorder. From this general argument it is already clear that the presence of impurities tends to suppress the conductivity in our holographic calculations.

\subsection{Random Dilaton: Charge Neutral Impurities}

Now we would like to study the DC conductivity (4.79) in more detail. First we would like to understand the effect of random values of the dilaton field $\phi$. As is clear from (2.32), a large value of the dilaton reduces the conductivity. For a large charge density $\rho z_{H}^{2} / R^{2}>>1$, we find

$$
\sigma_{D C} \simeq T_{p} \rho z_{H}^{2}\left\langle\frac{1}{\frac{1}{L} \int_{0}^{L} d x e^{\phi\left(z_{H}, x\right)}}\right\rangle
$$

In particular, if the region where the dilaton gets divergent has a small but nonzero volume, then the $\mathrm{DC}$ conductivity vanishes $\sigma_{D C}=0$ as is clear from (4.81). This holographically argues that the originally metallic system can be changed into an insulator in the presence of impurities. Notice that the right hand side of (4.80), which corresponds to a naive average, remains non-zero in this case.

In this way, we find that the dilatonic disorder effect highly reduces the DC conductivity as we expect from the standard knowledge for weakly coupled condensed matter 
systems. Notice that our results are obtained using holography with a supergravity approximation and thus the dual gauge theories are strongly coupled, which is usually very difficult to analyze in condensed matter physics.

One may worry that in the above argument, the divergent value of dilaton is important to realize insulators. However, we will later show in section 4.8 that it is still possible to obtain insulators in a different way without assuming singular backgrounds.

\subsection{Random Charge Density: Charged Impurities}

Next we study the effect of the random charge density $\rho(x)$, which induces random fluctuations of gauge potential in (4.58). To extract only this effect, we turn off the scalar fields $\phi=\varphi=0$ below. We start with a perturbative argument assuming that the randomness is small. Consider a fluctuation $\delta \beta(x)$ of the function $\beta(x)$ in (4.64) from the background charged solution (we revived $R$ dependence)

$$
\beta(x)=-\frac{\rho_{0}}{\sqrt{1+\frac{\rho_{0}^{2} z_{H}^{4}}{R^{4}}}}+\delta \beta(x),
$$

where $\rho_{0}$ is the original charge density. By evaluating the expression (4.56) perturbatively

$$
F\left(z_{H}, x\right)=\frac{1}{1-\frac{z_{H}^{4} \beta^{2}(x)}{R^{4}}}>1,
$$

in the end we find the leading correction due to the disorder effect

$$
\begin{aligned}
\Delta \sigma_{D C}= & \sigma_{D C}-T_{p} \sqrt{1+\rho_{0}^{2} z_{H}^{4}} \\
= & \frac{T_{p} z_{H}^{4}}{2 R^{4}}\left(\frac{1}{L} \int_{0}^{L} d x\left(1+\frac{\rho_{0}^{2} z_{H}^{4}}{R^{4}}\right)^{5 / 2}\left\langle(\delta \beta(x))^{2}\right\rangle\right) \\
& +\frac{T_{p} \rho_{0}^{2} z_{H}^{8}}{R^{8}}\left(1+\frac{\rho_{0}^{2} z_{H}^{4}}{R^{4}}\right)^{3 / 2}\left\langle\left(\frac{1}{L} \int_{0}^{L} d x \delta \beta(x)\right)^{2}\right\rangle \geq 0 .
\end{aligned}
$$

In this way, we find that the effect of the disordered charge density profile increases the conductivity when its random fluctuation is small. However, as we will explain this slightly non-standard behavior changes if the randomness increases or the temperature decreases. To see this, we come back to the expression (4.83). We can regard the amplitude of fluctuations of $\delta \beta(x)$ as the impurity density $\rho_{i m p}\left(<<\rho_{0}\right)$. The previous perturbation

breaks down when the temperature gets smaller than (remember the relation $T \sim \frac{1}{z_{H}}$ )

$$
T_{c} \sim \frac{\sqrt{\rho_{i m p}}}{R}
$$


Below this temperature, $\beta(x)$ is dominated by the random perturbation $\delta \beta(x)$ and there-

fore $\sigma_{D C}$ will decrease from the value (2.32) $\frac{\sigma_{D C}}{T_{p}} \sim \frac{\rho}{T_{c}^{2} R^{2}} \sim \frac{\rho}{\rho_{i m p}}>>1$ to $\frac{\sigma_{D C}}{T_{p}} \simeq 1$. Notice that $\frac{\sigma_{D C}}{T_{p}}$ is always greater than 1 as is clear from (4.83) and (4.79) because here we did not turn on the random fluctuations of closed string fields. These behavior is sketched in Fig. 12 .

This somewhat reminds us of what happens typically for the DC conductivity in systems with relativistic dispersion (e.g., graphene, quasi-particles in the two-dimensional $d$-wave superconductor,etc) where the disorder effect on the quantum transport can be interpreted in a twofold way. When the chemical potential is close to the Dirac point, disorder can increase the density of states (DOS), leading to enhancement of DC conductivity, which is however in competition with the diffusion constant diminished by disorder. As a consequence of the delicate balance between these, when the chemical potential is exactly at the Dirac point, a leading order effect of disorder potential that we see in, say, the self-consistent Born approximation or semiclassical analysis, often gives rise to the DC conductivity which is not affected by disorder. (However, as we increase disorder or as we lower temperature, we need to include higher order effects or quantum effects from disorder potential. In particular, these higher-order or non-perturbative effects in graphene include the Berry phase and topology associated to the single particle wavefunctions in graphene. Because of the topological reason, when the charge impurity potential does not change at atomic scales, disorder never be able to localize electrons but instead it increases the DC conductivity [56, 57, 58, 59. ) With interactions and with phonon bath which are included, together with disorder, in our holographic calculation in nonperturbative way, these competing effects are not in proportion any more and enhancing the conductivity.

In this setup we can have two mean free times (or relaxation times), each for the elastic scattering $\tau_{e l}$ and for the inelastic scattering $\tau_{i n}$. The former is due to the presence of impurities and we can estimate $\tau_{e l} \sim \frac{1}{\sqrt{\rho_{\text {imp }}}}$. On the other hand, the latter is due to the interactions mediated by gluons and is related to the temperature as $\tau_{\text {in }} \sim 1 / T$. Thus, as we decrease temperature, $\tau_{i n}$ exceeds $\tau_{e l}$ around the critical temperature $T_{c}$ (4.85).

\subsection{Random Holes on Horizon}

In the previous formulation, we took into account the random fluctuations of the scalar fields $\phi$ and $\varphi$ and the gauge potential $A_{t}$. One may think that we should also include those of the metric $g_{\mu \nu}$. In this paper we will not do this literally as calculations get highly complicated. Instead, we approximately incorporate the effect of large perturbations 


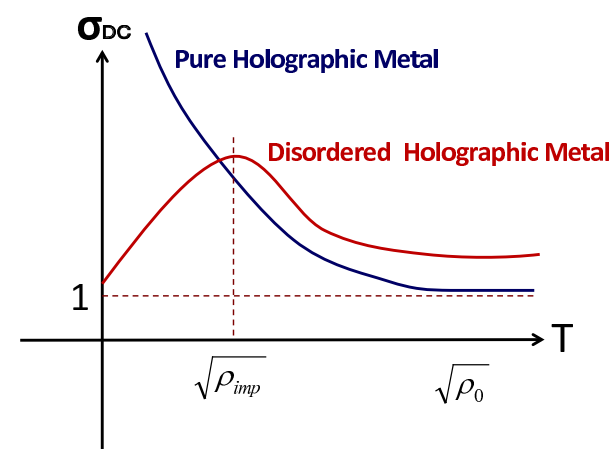

Figure 12: The schematic plot of the DC conductivity as a function of temperature in pure and disordered holographic metals with a random charge density. We simply set $T_{p}=R=1$.

which make small but many 'holes' on the horizon by locally replacing the black brane with a solitonic geometry, where the IR region is capped off (see Fig.13). As we mentioned, this introduces a mass gap locally and each hole is expected to describe holographically an impurity potential.

The situation can be compared with relativistic fermions subjected to "random mass" type disorder. It is described by the following single particle Hamiltonian:

$$
\mathcal{H}=-i \boldsymbol{\sigma} \cdot \boldsymbol{\partial}+m(x, y) \sigma_{z},
$$

where $\boldsymbol{\partial}=\left(\partial_{x}, \partial_{y}\right)$, and $\sigma_{x, y, z}$ denotes the $2 \times 2$ Pauli matrix; $m(x, y)$ represents a random mass term. Such a random mass Dirac problem was discussed in the context of e.g., the quantum Hall plateau transition, the random bond Ising model, and graphene (see, for example, [60], 61], 62 etc. and references therein). We should keep in mind, however, that our holographic calculation includes effects of interactions and heat bath on top of the disorder potential.

Instead of dealing with explicit metric, we will describe this situation qualitatively by changing the in-going boundary condition locally as

$$
A_{x}(w, x) \rightarrow a(x)\left(e^{i \omega\left(w-w_{\infty}\right)}+R(x) e^{-i \omega\left(w-w_{\infty}\right)}\right), \quad\left(w \rightarrow w_{\infty}\right)
$$

where $w_{\infty}$ is the IR cut off at which the tip of the AdS soliton located and $a(x)$ is an arbitrary function. The function $R(x)$ interpolates between $R(x)=0$ and $R(x)=1$, where $R(x)=0$ corresponds to the regular point where the impurity potential is negligible (or

\footnotetext{
${ }^{11}$ Numerical solutions of localized black holes inside a AdS soliton have been constructed in 63 .
} 


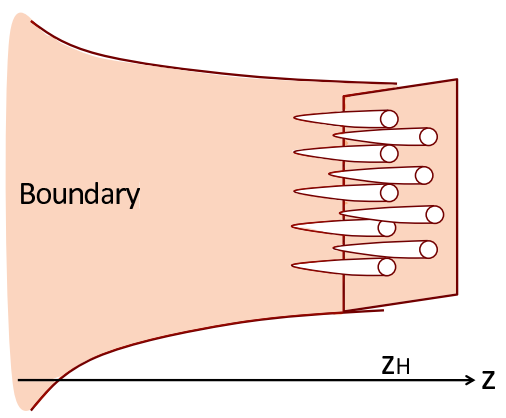

Figure 13: A sketch of holes on a black brane horizon which are dual to impurities in holographic metals.

equally the in-going boundary condition), and $R(x)=1$ corresponds to the center of impurity (or equally the Dirichlet boundary condition).

This randomly modified boundary condition is simply expressed as follows

$$
X(\infty, x)=i \omega \cdot \theta(x)
$$

where we defined $\theta(x)=\frac{1-R(x)}{1+R(x)}$. Points with $\theta(x)=0$ describe the impurities, while those with $\theta(x)=1$ do the metallic region. Notice that this is a description for low temperature systems and as we increase the temperature, the region with $\theta(x)=0$ will gradually disappear.

By employing this disordered boundary condition (4.88), the calculation of the conductivity can be done in the same way as we did in the previous section. The final formula (4.79) is simply replaced with

$$
\sigma_{D C}=T_{p}\left\langle\frac{1}{\frac{1}{L} \int_{0}^{L} d x \frac{1}{\theta(x) \sqrt{F\left(z_{H}, x\right)}}}\right\rangle .
$$

In this setup, we can realize the insulator $\sigma_{D C}=0$ by keeping the dilaton finite and staying with the regular solutions of supergravity. For example, if we assume the following profile

$$
\theta(x) \sqrt{F\left(z_{H}, x\right)}=\frac{(x-L / 2)^{2}+\epsilon^{2} a^{2}}{(x-L / 2)^{2}+a^{2}},
$$

\footnotetext{
${ }^{12}$ Strictly speaking we need to shift slightly the position from the horizon to impose (4.88). This is because if we literally impose (4.88), the order $\omega^{2}$ term of (4.66) gets divergent if $\theta(x) \neq 1$ when we integrate $w$ toward $\infty$ to find a solution of $A_{x}$. However, this divergence is artificial because we will not get the horizon geometry in the case of AdS soliton and we need to introduce another IR cut off $z_{i m p}>z_{H}$ which is dual to the mass gap due to the impurity. Thus we actually find that the $\omega^{2}$ term always remains finite and can be negligible in the DC limit $\omega \rightarrow 0$.
} 
then we find

$$
\sigma_{D C}=\frac{1}{1+\frac{\pi a}{\epsilon L}\left(1-\epsilon^{2}\right)},
$$

assuming $L \gg \epsilon$. Thus if we take the limit $\epsilon=0$, then $\sigma_{D C}$ vanishes. More generally, if the function $\theta(x)$ has at least one generic zero $x=x_{0}$ i.e. $\theta\left(x_{0}\right)=0$ with $\theta(x) \propto\left(x-x_{0}\right)^{2}$ in the limit $x \rightarrow x_{0}$, then the DC conductivity vanishes. This shows that even a single impurity can turn a metal into an insulator in $1+1$ dimensions.

It is known that a similar thing happens in the Tomonaga-Luttinger liquid when there are predominantly repulsive electron-electron interactions 64, 65]. In this case, the local potential is a relevant operator in the RG sense and at low energies (at low temperature), the single impurity effectively cuts the systems into two separate parts.

Before we go on, we would like to remember again that to obtain the result (4.89), we assumed codimension one impurities. Therefore, more naturally, this corresponds to point-like impurities in $1+1$ dimensional system described by $\mathrm{AdS}_{3} / \mathrm{CFT}_{2}$. In this case the DC conductivity is given by a similar formula

$$
\sigma_{D C}=T_{p} z_{H}\left\langle\frac{1}{\frac{1}{L} \int_{0}^{L} d x \frac{1}{\theta(x) \sqrt{F\left(z_{H}, x\right)}}}\right\rangle .
$$

\subsection{Codimension Two Randomness}

So far our holographic calculations are restricted to the codimension one impurities by assuming that the randomness only depends on $x$ but not on $y$. Even though this is enough to describe $1+1$ dimensional disordered systems, for realistic setups of $2+1$ dimensional systems, usually the impurities are point-like (i.e. codimension two) and thus we need to further incorporate the $y$ dependence.

If we allow both $F$ and $\theta$ to depend on both $x$ and $y$, the equation of motion gets highly complicated. Thus here we assume that $F=F(z)$ is a constant function of $x$ and $y$, while $\theta=\theta(x, y)$ is a random function. This means that we ignore fluctuations of the gauge field. Thus our result below can give full contributions only when the charge density is vanishing $\rho=0$. In this case, the equations of motion of the gauge fields take the following form (each comes from $x, y$ and $z$ component) to the leading order

$$
\begin{aligned}
& \partial_{w}\left(\sqrt{F} \partial_{w} A_{x}\right)=\partial_{y}(\cdots), \\
& \partial_{w}\left(\sqrt{F} \partial_{w} A_{y}\right)=\partial_{x}(\cdots), \\
& \partial_{x}\left(\sqrt{F} \partial_{w} A_{x}\right)+\partial_{y}\left(\sqrt{F} \partial_{w} A_{y}\right)=0,
\end{aligned}
$$


where $(\cdots)$ represent certain functionals of the gauge field whose details are not important. Assuming an appropriate boundary condition in $y$ direction as before, we can see from (4.93) and (4.95) that

$$
\sigma_{D C}=\frac{T_{p}}{L} \int_{0}^{L} d y \sqrt{F(z)} \frac{\partial_{w} A_{x}(z, x, y)}{i \omega},
$$

does not depend on $x$ and $w$ and therefore coincides with the DC conductivity.

By generalizing (4.72), we impose the boundary behavior

$$
\begin{aligned}
& A_{t}(0, x, y)=i \omega p(x, y)+O\left(\omega^{2}\right), \\
& A_{x}(0, x, y)=1-\partial_{x} p(x, y)+O\left(\omega^{2}\right), \\
& A_{y}(0, x, y)=-\partial_{y} p(x, y)+O\left(\omega^{2}\right), \\
& X\left(z_{H}, x, y\right)=i \omega \theta(x, y) .
\end{aligned}
$$

Then, the equation (4.95) evaluated at the horizon leads to

$$
\partial_{x}\left[\theta(x, y)\left(1-\partial_{x} p(x, y)\right)\right]-\partial_{y}\left[\theta(x, y) \partial_{y} p(x, y)\right]=0 .
$$

Moreover, we need to impose the boundary condition such that the average of the gradient of the charge density is vanishing as we did before in (4.78). One choice will be to require

$$
p(x, y)=0
$$

on the boundary of the region with the linear size L. After solving (4.98) with this boundary condition, the DC conductivity is finally obtained from (4.96) setting $z=z_{H}$ :

$$
\sigma_{D C}=\frac{T_{p} \sqrt{F\left(z_{H}\right)}}{L} \int_{0}^{L} d y \theta(x, y)\left(1-\partial_{x} p(x, y)\right) .
$$

For simplicity, we suppress the coefficient $T_{p} \sqrt{F\left(z_{H}\right)}$ below, by setting this to be one.

In general, we cannot expect analytical solutions to the present problem (4.98) and (4.99). However, it is helpful to rewrite (4.98) into a form like Schrodinger problem by defining

$$
\varphi(x, y)=\sqrt{\theta(x, y)} p(x, y) .
$$

This satisfies the following Schrodinger equation with a source:

$$
-\left(\partial_{x}^{2}+\partial_{y}^{2}\right) \varphi+V(x, y) \varphi=W(x, y),
$$

where

$$
\begin{aligned}
& V(x, y)=\frac{\left(\partial_{x}^{2}+\partial_{y}^{2}\right) \theta}{2 \theta}-\frac{\left(\partial_{x} \theta\right)^{2}+\left(\partial_{y} \theta\right)^{2}}{4 \theta^{2}} \\
& W(x, y)=-\frac{\partial_{x} \theta}{\sqrt{\theta}} .
\end{aligned}
$$


As an example, we model a single impurity situated at $x=y=0$ by the profile

$$
\theta(x, y)=\frac{r^{2}}{r^{2}+1}
$$

where we employ the polar coordinate $x=r \sin (s)$ and $y=r \cos (s)$. Also we obtain

$$
V(r)=\frac{1-2 r^{2}}{r^{2}\left(1+r^{2}\right)^{2}}, \quad W(r, s)=-\frac{2 \sin (s)}{\left(1+r^{2}\right)^{3 / 2}} .
$$

We consider the system which is given by a disk with radius $L$ and the boundary condition (4.99) leads to

$$
\varphi(r=L)=0 .
$$

We can assume the following form of solutions

$$
\varphi(x, y)=\varphi(r) \sin (s)
$$

The equation (4.102) is written explicitly as

$$
-\partial_{r}^{2} \varphi-\frac{\partial_{r} \varphi}{r}+\left(V(r)+\frac{1}{r^{2}}\right) \varphi=-\frac{2}{\left(1+r^{2}\right)^{3 / 2}} .
$$

This equation with the smoothness condition at $r=0$ requires the behavior $\varphi(r) \propto r^{\sqrt{2}}$ and thus $p(x, y)$ behaves like

$$
p(x, y)=(\text { const. }) \cdot r^{\sqrt{2}-1} \sin (s), \quad(r \rightarrow 0) .
$$

The conductivity is expressed as

$$
\sigma_{D C}=\sigma_{0}+\Delta \sigma
$$

where

$$
\begin{aligned}
& \sigma_{0}=\frac{2}{L^{2}} \int_{0}^{L} d r r \theta(r), \\
& \Delta \sigma=-\frac{1}{L^{2}} \int_{0}^{L} d r r \theta(r)\left(\frac{\varphi(r)}{r \sqrt{\theta(r)}}+\frac{\partial}{\partial r}\left(\frac{\varphi(r)}{\sqrt{\theta(r)}}\right)\right) .
\end{aligned}
$$

We numerically confirmed that the difference $\Delta \sigma$ between the true DC conductivity $\sigma_{D C}$ and its naive value $\sigma_{0}$ is negative for any choice of $L$ as plotted in Fig. 14 and this property is similar to the previous codimension one case. As it shows, it approaches to $\Delta \sigma \simeq-\frac{0.831}{L^{2}}$ in the $L \rightarrow \infty$ limit. 


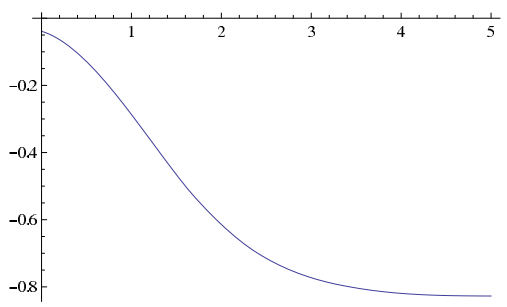

Figure 14: The value of $L^{2} \cdot \Delta \sigma$ defined in (4.111) versus the value of $\log L$.

Notice that in this holographic calculation for the codimension two impurity, $\Delta \sigma$ cannot be order one as opposed to the previous codimension one case. Therefore in $2+1$ dimension we can conclude that a single or finite number of impurities cannot make a metal into an insulator, as expected in condensed matter physics.

Another simple example will be the case where $\theta$ depends only[3 on $y$. Because the source term $W$ in (4.102) vanishes, we can set $\varphi=p=0$. Thus we find that the naive conductivity coincides with $\sigma_{D C}$

$$
\sigma_{D C}=\frac{1}{L} \int_{0}^{L} d y \theta(y) .
$$

This shows that $\sigma_{D C}$ is non-vanishing unless $\theta(y)=0$ identically.

One of the most interesting questions in this system will be whether it shows a metalinsulator phase transition as we increases the number of impurities. Even though we need a full numerical analysis to answer this question completely, our previous analysis suggested that the conductivity becomes zero only if there is no connected path between $x=0$ and $x=L$ on which $\theta$ is non-vanishing. For example, if $\theta$ vanishes at $x=x_{0}$ for any values of $y$, the conductivity vanishes as we showed in the previous section. For schematic examples, see the Fig. 15.

Finally it is intriguing to notice that in the presence of random distributions of $\theta(x, y)$, the potential $V$ becomes a random function of $x$ and $y$ and our problem to solve (4.102) looks similar to the problem of Anderson localization, which is also described by a random potential.

\footnotetext{
${ }^{13}$ Note that if $\theta$ depends only on $x$, the system is reduced to the system discussed in the previous subsection.
} 

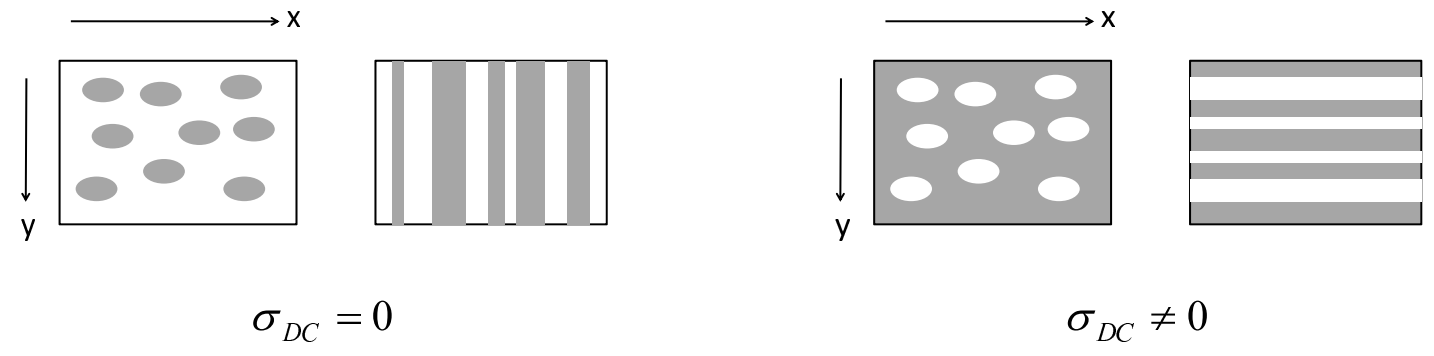

Figure 15: Schematic examples of the holographic conductivity. The white regions represent the ones where $\theta=0$. The gray regions are the ones where $\theta>0$. The left two figures correspond to an insulator, while the right ones to a metal.

\section{Conclusions and Discussions}

In this paper we studied the holographic conductivity in various probe brane systems via the AdS/CFT correspondence. In the first half of this paper, we analyzed rather general setups of probe D-branes which are dual to $2+1$ dimensional systems. They are flavor or defect sectors of gauge theories, which are coupled to gluons in the same or higher dimensions. Our formalism incorporates the effect of dilaton which depends on the radial coordinate of the AdS. We present a systematic analysis of AC and DC conductivity by relating this to the Schrodinger problem. As one of the examples, we found that a known dilatonic solution, which can be embedded in string theory, leads to a peculiar pseudo-gap of the form $\operatorname{Re} \sigma(\omega) \sim \omega^{1 / 3}$ in addition to the delta functional Drude peak at zero temperature. In order to realize insulators, we somehow need to remove the Drude peak. Since the DC conductivity depends on the value of dilaton at the horizon, we noted that a system with a divergent dilaton can be regarded as a holographic insulator, which resembles Mott insulators. We confirmed this interpretation by calculating AC conductivity numerically and found that we can realize various insulators by choosing the dilaton profile appropriately.

In the latter half, we concentrated on the main issue of this paper. We studied the holographic conductivity in disordered systems. In particular, we again employed the probe D-brane setups because they allow us to calculate the effect of randomness in the classical analysis and because we can ignore complicated backreactions. We consider three important origins of the disorder. Two of them are closed string fields: dilaton and the metric. We treat the metric fluctuations as holes on the horizon, assuming a low temperature region. The other is an open string field: the $U(1)$ gauge field on the probe D-brane, which corresponds to the random charged density. We showed that the 
dilaton and metric randomness can reduce the DC conductivity and can finally take it to be zero. On the other hand, the disordered charge density increases the conductivity at high temperature and as the temperature goes down, its effect changes the sign and eventually drives it to a certain small but non-zero value. We also managed to obtain an analytical formula of DC conductivity for the codimension one randomness. In more general cases, we gave a perturbative estimation in the presence of the disordered closed string fields.

Finally, one of the most intriguing questions will be whether our holographic analysis dual to strongly coupled disordered systems leads to a phenomenon similar to Anderson localization, which has been usually derived for weakly coupled electrons. For example, let us choose the characteristic values at finite temperature $T \sim 1 / z_{H}$ in the profile (4.90) as $a \sim T^{-1}$ and $L \sim T^{-1}$. Assuming that the disorder effect is very small at high enough temperature, we can treat $\delta \equiv 1-\epsilon^{2}$ as a very small parameter $\delta \ll 1$. In the $\mathrm{AdS}_{3}$ case dual to one dimensional systems, the DC conductivity (4.92) is perturbatively estimated to be $\sigma_{D C}=\sigma_{D C}^{\text {pure }}-C \cdot \frac{\delta}{T}+O\left(\delta^{2}\right)$, where $\sigma_{D C}^{\text {pure }}$ is the conductivity without disorder and $C$ is a positive numerical constant. This dependence on the temperature seems to be consistent with the known formula in weak localization [24, 25] for one dimensional systems. However, a similar analysis in $\mathrm{AdS}_{4}$ case does not seem to reproduce the known logarithmic dependence on the temperature. Therefore we cannot argue that our holographic analysis in supergravity approximation, which is dual to the strong coupling limit and large N limit of CFT, support the Anderson localization. We hope we can come back to more details in future publications.

Acknowledgments We are grateful to Hong Liu and Elias Kiritsis for useful conversations. SR is supported by Center for Condensed Matter Theory at University of California, Berkeley. TT and TU are supported by World Premier International Research Center Initiative (WPI Initiative), MEXT, Japan. SR and TT would like to thank the winter conference "Strongly Correlated Systems and Gauge/Gravity Duality," held in 2011 at Aspen center for physics, where a part of this work was completed. TT is grateful to the workshop "AdS/CM duality and other approaches," held in 2010 at KITPC, the Chinese Academy of Sciences, where an initial stage of this work has been progressed. The work of TT is also supported in part by JSPS Grant-in-Aid for Scientific Research No.20740132, and by JSPS Grant-in-Aid for Creative Scientific Research No. 19 GS0219. 


\section{A Sum Rule}

Since the sum rule is useful to understand the general behavior of the conductivity, here we would like to summarize it. The general aspect of sum rules in AdS/CFT has been considered in (66]). Here we would like to concentrate on that of conductivity in the $2+1$ dimensional critical system and study its consequence.

If the retarded Green function $G_{R}(\omega)$ is holomorphic for $\operatorname{Im} \omega \geq 0$ and it satisfies $\lim _{|\omega| \rightarrow 0} G_{R}(\omega) \rightarrow 0$ for $\operatorname{Im} \omega \geq 0$, then

$$
G_{R}(0)=\lim _{\epsilon \rightarrow 0} \int_{-\infty}^{\infty} \frac{d \omega}{\pi} \frac{\operatorname{Im} G_{R}(\omega)}{\omega-i \epsilon}
$$

Since $\operatorname{Im} G_{R}(\omega)$ is an odd function of $\omega$, we can replace the integral in (A.114) with

$$
G_{R}(0)=2 P \int_{0}^{\infty} \frac{d \omega}{\pi} \frac{\operatorname{Im} G_{R}(\omega)}{\omega} .
$$

Now we remember the relation in linear response theory

$$
\sigma(\omega)=\frac{\left\langle J_{x}(\omega) J_{x}(0)\right\rangle_{R}}{\omega} .
$$

In $2+1$ dimensional systems which are critical in the UV limit, we know

$$
\sigma(\omega) \rightarrow \sigma_{0} \quad(\omega \rightarrow \infty)
$$

where $\sigma_{0}$ is a constant and is given by $T_{p}$ in our holographic calculation. To maintain the damping condition, we define

$$
G_{R}(\omega)=-i\left\langle J_{x}(\omega) J_{x}(0)\right\rangle_{R}+i \sigma_{0} \omega
$$

and we apply the sum rule (A.114). When $\sigma(\omega)$ is a smooth function, this leads to the sum rule of the conductivity

$$
\int_{0}^{\infty} d \omega\left(\operatorname{Re} \sigma(\omega)-\sigma_{0}\right)=0
$$

because we can set $G_{R}(0)=0$ (or equally there is no pole in $\operatorname{Im} \sigma(\omega)$ ).

When there is a delta function Drude peak, if we define $n$ by $\sigma(\omega) \rightarrow \frac{i n}{\omega}$ in the limit $\omega \rightarrow 0$, we obtain the following modified sum rule

$$
\int_{0+}^{\infty} d \omega\left(\operatorname{Re} \sigma(\omega)-\sigma_{0}\right)=-\frac{\pi}{2} n
$$

where notice that the integral does not include the contribution from the delta functional part. 
It will be instructive to compare the above with the standard one in non-relativistic condensed matter systems (see e.g.[67]). The latter is given by

$$
\int_{0}^{\infty} d \omega \operatorname{Re} \sigma(\omega)=2 \pi n_{0}>0
$$

where $n_{0}$ is proportional to the electron density. This is obtained by assuming the behavior of plasma oscillation

$$
\sigma(\omega) \rightarrow \frac{i n_{0}}{\omega}, \quad(\omega \rightarrow \infty)
$$

The difference of the UV behavior between (A.116) and (A.121) is crucial to have two different sum rules.

\section{References}

[1] J. M. Maldacena, "The large N limit of superconformal field theories and supergravity," Adv. Theor. Math. Phys. 2 (1998) 231 [Int. J. Theor. Phys. 38 (1999) 1113] [arXiv:hep-th/9711200];

[2] S. S. Gubser, I. R. Klebanov and A. M. Polyakov, "Gauge theory correlators from non-critical string theory," Phys. Lett. B 428, 105 (1998) [arXiv:hep-th/9802109].

[3] E. Witten, "Anti-de Sitter space and holography," Adv. Theor. Math. Phys. 2, 253 (1998) [arXiv:hep-th/9802150].

[4] O. Aharony, S. S. Gubser, J. M. Maldacena, H. Ooguri and Y. Oz, "Large N field theories, string theory and gravity," Phys. Rept. 323, 183 (2000) [arXiv:hep-th/9905111].

[5] S. A. Hartnoll, "Lectures on holographic methods for condensed matter physics," Class. Quant. Grav. 26 (2009) 224002 [arXiv:0903.3246 [hep-th]].

[6] C. P. Herzog, "Lectures on Holographic Superfluidity and Superconductivity," J. Phys. A 42, 343001 (2009) [arXiv:0904.1975 [hep-th]];

[7] T. Nishioka, S. Ryu and T. Takayanagi, "Holographic Entanglement Entropy: An Overview," J. Phys. A 42 (2009) 504008 [arXiv:0905.0932 [hep-th]].

[8] J. McGreevy, "Holographic duality with a view toward many-body physics," Adv. High Energy Phys. 2010 (2010) 723105 [arXiv:0909.0518 [hep-th]].

[9] S. Sachdev, "Condensed matter and AdS/CFT," arXiv:1002.2947 [hep-th].

[10] S. Ryu and T. Takayanagi, "Holographic derivation of entanglement entropy from AdS/CFT," Phys. Rev. Lett. 96 (2006) 181602 [arXiv:hep-th/0603001]; "Aspects of holographic entanglement entropy," JHEP 0608 (2006) 045 [arXiv:hep-th/0605073].

[11] S. S. Gubser, "Breaking an Abelian gauge symmetry near a black hole horizon," Phys. Rev. D 78 (2008) 065034 [arXiv:0801.2977 [hep-th]]. 
[12] S. A. Hartnoll, C. P. Herzog, G. T. Horowitz, "Building a Holographic Superconductor," Phys. Rev. Lett. 101 (2008) 031601. [arXiv:0803.3295 [hep-th]]; "Holographic Superconductors," JHEP 0812 (2008) 015 [arXiv:0810.1563 [hep-th]].

[13] D. T. Son, "Toward an AdS/cold atoms correspondence: a geometric realization of the Schroedinger symmetry," Phys. Rev. D 78 (2008) 046003 [arXiv:0804.3972 [hep-th]]; K. Balasubramanian and J. McGreevy, "Gravity duals for non-relativistic CFTs," Phys. Rev. Lett. 101, 061601 (2008) [arXiv:0804.4053 [hep-th]].

[14] S. Kachru, X. Liu, M. Mulligan, "Gravity Duals of Lifshitz-like Fixed Points," Phys. Rev. D78 (2008) 106005. [arXiv:0808.1725 [hep-th]].

[15] K. Goldstein, S. Kachru, S. Prakash and S. P. Trivedi, "Holography of Charged Dilaton Black Holes," JHEP 1008 (2010) 078. [arXiv:0911.3586 [hep-th]]; K. Goldstein, N. Tizuka, S. Kachru, S. Prakash, S. P. Trivedi and A. Westphal, "Holography of Dyonic Dilaton Black Branes," JHEP 1010 (2010) 027. [arXiv:1007.2490 [hep-th]].

[16] H. Liu, J. McGreevy and D. Vegh, "Non-Fermi liquids from holography," arXiv:0903.2477 [hep-th]; T. Faulkner, H. Liu, J. McGreevy and D. Vegh, "Emergent quantum criticality, Fermi surfaces, and AdS2," arXiv:0907.2694 [hep-th].

[17] For reviews and references see T. Faulkner, N. Iqbal, H. Liu, J. McGreevy and D. Vegh, "From black holes to strange metals," arXiv:1003.1728 [hep-th]; J. McGreevy, "Holographic duality with a view toward many-body physics," arXiv:0909.0518 [hep-th];

[18] J. L. Davis, P. Kraus, A. Shah, "Gravity Dual of a Quantum Hall Plateau Transition," JHEP 0811 (2008) 020. [arXiv:0809.1876 [hep-th]]; J. Alanen, E. KeskiVakkuri, P. Kraus and V. Suur-Uski, "AC Transport at Holographic Quantum Hall Transitions," JHEP 0911 (2009) 014. [arXiv:0905.4538 [hep-th]].

[19] M. Fujita, W. Li, S. Ryu and T. Takayanagi, "Fractional Quantum Hall Effect via Holography: Chern-Simons, Edge States, and Hierarchy," JHEP 0906 (2009) 066. [arXiv:0901.0924 [hep-th]]; Y. Hikida, W. Li, T. Takayanagi, "ABJM with Flavors and FQHE," JHEP 0907 (2009) 065. [arXiv:0903.2194 [hep-th]].

[20] O. Bergman, N. Jokela, G. Lifschytz and M. Lippert, "Quantum Hall Effect in a Holographic Model," JHEP 1010 (2010) 063. [arXiv:1003.4965 [hep-th]].

[21] S. Ryu and T. Takayanagi, "Topological Insulators and Superconductors from Dbranes," Phys. Lett. B 693 (2010) 175 [arXiv:1001.0763 [hep-th]]; "Topological Insulators and Superconductors from String Theory," Phys. Rev. D 82 (2010) 086014 [arXiv:1007.4234 [hep-th]].

[22] C. Hoyos-Badajoz, K. Jensen and A. Karch, "A Holographic Fractional Topological Insulator," Phys. Rev. D 82 (2010) 086001 [arXiv:1007.3253 [hep-th]]; A. Karch, J. Maciejko and T. Takayanagi, "Holographic fractional topological insulators in 2+1 and 1+1 dimensions," Phys. Rev. D 82 (2010) 126003 [arXiv:1009.2991 [hep-th]].

[23] P. W. Anderson, "Absence of Diffusion in Certain Random Lattices," Phys. Rev. 109, 1492, 1958. 
[24] E. Abrahams, P. W. Anderson, D. C. Licciardello, "Scaling Theory of Localization: Absence of Quantum Diffusion in Two Dimensions," T. V. Ramakrishnan, Phys. Rev. Lett, 42, 673, 1979.

[25] P. A. Lee and T. V. Ramakrishnan, "Disordered electronic systems," Rev. Mod. Phys. 57, 287 (1985).

[26] D. Belitz and T. R. Kirkpatrick, "The Anderson-Mott transition," Rev. Mod. Phys. 66, 261 (1994).

[27] F. Evers and A. D. Mirlin, "Anderson Transitions," Rev. Mod. Phys. 80, 1355 (2008).

[28] S. V. Kravchenko and M. P. Sarachik, "Metal-insulator transition in two-dimensional electron systems," Rep. Prog. Phys. 67, 1 (2004); A. Punnoose and A. M. Finkel'stein, Science 310, 289 (2005).

[29] S. Das Sarma, Shaffique Adam, E. H. Hwang, Enrico Rossi, "Electronic transport in two dimensional graphene," [arXiv:1003.4731 [pdf, other]].

[30] S. A. Hartnoll and C. P. Herzog, "Impure AdS/CFT," Phys. Rev. D 77 (2008) 106009 [arXiv:0801.1693 [hep-th]].

[31] M. Fujita, Y. Hikida, S. Ryu and T. Takayanagi, "Disordered Systems and the Replica Method in AdS/CFT," JHEP 0812 (2008) 065. [arXiv:0810.5394 [hep-th]].

[32] S. Kachru, A. Karch and S. Yaida, "Holographic Lattices, Dimers, and Glasses," Phys. Rev. D 81 (2010) 026007 [arXiv:0909.2639 [hep-th]]; "Adventures in Holographic Dimer Models," arXiv:1009.3268 [hep-th].

[33] For a recent study of functional renormalization group in disordered electron systems, see, e.g., Matthew S. Foster, Shinsei Ryu, Andreas W. W. Ludwig, "Termination of typical wavefunction multifractal spectra at the Anderson metal-insulator transition: Field theory description using the functional renormalization group," Phys. Rev. B 80, 075101 (2009); see also Viewpoint in Physics 2, 66 (2009).

[34] A. Adams and S. Yaida, "Disordered Holographic Systems I: Functional Renormalization," arXiv:1102.2892 [hep-th].

[35] L. Y. Hung and Y. Shang, "On 1-loop diagrams in AdS space," Phys. Rev. D 83 (2011) 024029 [arXiv:1007.2653 [hep-th]].

[36] A. Karch and E. Katz, "Adding flavor to AdS/CFT," JHEP 0206 (2002) 043 [arXiv:hep-th/0205236].

[37] A. Karch and A. O'Bannon, "Metallic AdS/CFT," JHEP 0709 (2007) 024 [arXiv:0705.3870 [hep-th]].

[38] A. Karch and L. Randall, "Localized gravity in string theory," Phys. Rev. Lett. 87 (2001) 061601 [arXiv:hep-th/0105108]; "Open and closed string interpretation of SUSY CFT's on branes with boundaries," JHEP 0106 (2001) 063 [arXiv:hepth/0105132]. 
[39] R. C. Myers and M. C. Wapler, "Transport Properties of Holographic Defects," JHEP 0812 (2008) 115 [arXiv:0811.0480 [hep-th]].

[40] S. A. Hartnoll, J. Polchinski, E. Silverstein and D. Tong, "Towards strange metallic holography," arXiv:0912.1061 [hep-th].

[41] C. -M. Chen, D. -W. Pang, "Holography of Charged Dilaton Black Holes in General Dimensions," JHEP 1006 (2010) 093. [arXiv:1003.5064 [hep-th]].

[42] S. R. Das, T. Nishioka and T. Takayanagi, "Probe Branes, Time-dependent Couplings and Thermalization in AdS/CFT," JHEP 1007 (2010) 071 [arXiv:1005.3348 [hepth]].

[43] C. Charmousis, B. Gouteraux, B. S. Kim, E. Kiritsis and R. Meyer, "Effective Holographic Theories for low-temperature condensed matter systems," JHEP 1011 (2010) 151. [arXiv:1005.4690 [hep-th]].

[44] C. Hoyos-Badajoz, A. O'Bannon, J. M. S. Wu, "Zero Sound in Strange Metallic Holography," JHEP 1009 (2010) 086. [arXiv:1007.0590 [hep-th]].

[45] M. Imada, A. Fujimori and Y. Tokura, "Metal-insulator transitions," Rev. Mod. Phys. 70, 1039 (1998).

[46] U. Gursoy, E. Kiritsis, L. Mazzanti, F. Nitti, "Holography and Thermodynamics of 5D Dilaton-gravity", [arXiv:0812.0792 [hep-th]].

[47] T. Azeyanagi, W. Li, T. Takayanagi, "On String Theory Duals of Lifshitz-like Fixed Points," JHEP 0906 (2009) 084. [arXiv:0905.0688 [hep-th]].

[48] D. T. Son and A. O. Starinets, "Minkowski-space correlators in AdS/CFT correspondence: Recipe and applications," JHEP 0209 (2002) 042 [arXiv:hep-th/0205051].

[49] M. Ammon, J. Erdmenger, M. Kaminski and P. Kerner, "Superconductivity from gauge/gravity duality with flavor," Phys. Lett. B680 (2009) 516-520. [arXiv:0810.2316 [hep-th]].

[50] T. Nishioka, S. Ryu, T. Takayanagi, "Holographic Superconductor/Insulator Transition at Zero Temperature," JHEP 1003 (2010) 131. [arXiv:0911.0962 [hep-th]].

[51] K. Balasubramanian and J. McGreevy, "The particle number in Galilean holography," JHEP 1101 (2011) 137 [arXiv:1007.2184 [hep-th]].

[52] M. Edalati, R. G. Leigh and P. W. Phillips, "Dynamically Generated Gap from Holography: Mottness from a Black Hole," arXiv:1010.3238 [hep-th].

[53] E. Witten, "Anti-de Sitter space, thermal phase transition, and confinement in gauge theories," Adv. Theor. Math. Phys. 2 (1998) 505 [arXiv:hep-th/9803131].

[54] G. T. Horowitz and R. C. Myers, "The AdS/CFT Correspondence and a New Positive Energy Conjecture for General Relativity," Phys. Rev. D 59 (1998) 026005 [arXiv:hep-th/9808079]. 
[55] H. U. Baranger, A. D. Stone, "Electrical linear-response theory in an arbitrary magnetic field: A new Fermi-surface formation," Phys. Rev. bf B 40, 8169 (1989); S. Xiong, N. Read, A. D. Stone, "Mesoscopic conductance and its fluctuations at non-zero Hall angle ," Phys. Rev. bf B 56, 3982 (1997), [arXiv:cond-mat/9701077].

[56] P. M. Ostrovsky, I. V. Gornyi, and A. D. Mirlin, "Quantum criticality and minimal conductivity in graphene with long-range disorder," Phys. Rev. Lett. 98, 256801 (2007), [arXiv:cond-mat/0702115].

[57] Shinsei Ryu, Christopher Mudry, Hideaki Obuse, Akira Furusaki, "Z2 topological term, the global anomaly, and the two-dimensional symplectic symmetry class of Anderson localization," Phys. Rev. Lett. 99, 116601 (2007).

[58] J. H. Bardarson, J. Tworzydło, P. W. Brouwer, and C. W. J. Beenakker, "Demonstration of one-parameter scaling at the Dirac point in graphene," Phys. Rev. Lett. 99, 106801 (2007).

[59] Kentaro Nomura, Mikito Koshino, Shinsei Ryu, "Topological delocalization of twodimensional massless Dirac fermions," Phys. Rev. Lett. 99, 146806 (2007).

[60] A. W. W. Ludwig, M. P. A. Fisher, R. Shankar and G. Grinstein, "Integer quantum Hall transition: An alternative approach and exact results," Phys. Rev. B 50, 7526 (1994).

[61] S. Cho and M. P. A. Fisher, "Conductance fluctuations at the integer quantum Hall plateau transition," Phys. Rev. B 55, 1673 (1997).

[62] Kentaro Nomura, Shinsei Ryu, Mikito Koshino, Christopher Mudry, Akira Furusaki, "Quantum Hall Effect of Massless Dirac Fermions in a Vanishing Magnetic Field," Phys. Rev. Lett. 100, 246806 (2008).

[63] O. Aharony, S. Minwalla and T. Wiseman, "Plasma-balls in large N gauge theories and localized black holes," Class. Quant. Grav. 23 (2006) 2171 [arXiv:hepth/0507219].

[64] C. L. Kane and M. P. A. Fisher, "Transport in a one-channel Luttinger liquid," Phys. Rev. Lett. 68, 1220 (1992).

[65] A. Furusaki and N. Nagaosa, "Single-barrier problem and Anderson localization in a one-dimensional interacting electron system,"

Phys. Rev. B 47, 4631 (1993).

[66] D. R. Gulotta, C. P. Herzog, M. Kaminski, "Sum Rules from an Extra Dimension," JHEP 1101 (2011) 148. [arXiv:1010.4806 [hep-th]].

[67] G. D. Mahan, Many-Particle Physics, third eidition, 2000, Kluwer Academic/Plenum Publishers. 